Juan Manuel Candelo Viafara*

Universidad del Valle Cali, Colombia

Recibido: 18 de mayo de 2018 Concepto de evaluación: 27 de junio de 2018 Aprobado: 27 de junio de 2018

Artículo de investigación (C) 2018 Universidad Católica de Colombia. Facultad de Ciencias Económicas y Administrativas. Todos los derechos reservados

* Administrador de empresas, Magíster en Economía Aplicada; docente de la Universidad del Valle, sede Buga, Colombia. Correo electrónico: juan.candelo@ correounivalle.edu.co. https:// orcid.org/0000-0002-4152-5597
Finanz. polit. econ., ISSN: 2248-6046, Vol. 10, N. ${ }^{\circ} 2$, julio-diciembre, 2018, pp. 403-436

http://dx.doi.org/10.14718/revfinanzpolitecon.2018.10.2.9

\section{Impactos indirectos de la tasa de cambio y los precios del petróleo en una economía no petrolera: aproximaciones VECM y VAR para el Valle del Cauca, Colombia*}

\section{RESUMEN}

Este artículo forma parte de literatura poco explorada y busca identificar la relación entre el precio del petróleo y la tasa de cambio real en una economía pequeña, abierta y regional del departamento del Valle del Cauca, en Colombia. Se utilizan vectores de corrección de errores (VECM) y vectores autorregresivos (VAR) con datos mensuales entre 2001 y 2015. Los resultados identifican que un impulso de la tasa de cambio real genera impactos positivos, mientras que un impulso del precio del petróleo origina efectos negativos y asimétricos en la economía, debido a la pérdida de competitividad de la industria y al deterioro del consumo.

Palabras clave: cointegración, enfermedad holandesa, precios del petróleo, tasa de cambio real, VAR, VECM.

JEL: C32, F14, E17, E32, Q43

Indirect impacts of the exchange rate and petroleum prices in a non-oil economy: VECM and VAR approaches for the Valle del Cauca, Colombia

\section{ABSTRACT}

This article is part of a little explored literature, seeking to identify the relationship between the price of petroleum and the real exchange rate in a small, open, and regional economy in the department of Valle del Cauca, Colombia. A Vector Error Correction Model (VECM) and a Vector Autoregression Model

Este artículo forma parte del trabajo de grado para optar al título de magíster en Economía Aplicada de la Universidad del Valle. Agradecimientos especiales a la profesora Lya Paola sierra, Ph. D. en Economía de la Universidad Autónoma de Madrid; y a Julio Escobar Potes, director del Centro Regional de Estudios Económicos del Banco de la República, Cali. 
(VAR) are used with monthly data from 2001 to 2015. The results show that a boost in real exchange rate generates positive impacts on the economy and a boost in the price of petroleum generates negative and asymmetric effects in the economy. This is due to a negative effect on industry competitiveness and the wear and tear of consumption.

Keywords: Petroleum prices, real exchange rate, Dutch disease, cointegration, VECM, VAR.

\section{Impactos indiretos da taxa de câmbio e dos preços do petróleo numa economia não petroleira: aproximações VECM e VAR para o Valle del Cauca, Colômbia}

\section{RESUMO}

Este artigo faz parte de literatura pouco explorada e busca identificar a relação entre o preço do petróleo e a taxa de câmbio real numa economia pequena, aberta e regional do estado do Valle del Cauca, na Colômbia. São utilizados vetores de correção de erros (VECM) e vetores autorregresivos (VAR) com dados mensais entre 2001 e 2015. Os resultados identificam que um impulso da taxa de câmbio real gera impactos positivos, enquanto um impulso do preço do petróleo origina efeitos negativos e assimétricos na economia, devido à perda de concorrência da indústria e à deterioração do consumo.

Palavras-chave: cointegração, doença holandesa, preços do petróleo, taxa de câmbio real, VAR, VECM. 


\section{INTRODUCCIÓN}

El Valle del Cauca aportó al producto interno bruto (PIB) nacional un $10,0 \%$ para 2016 , participación importante que lo posiciona en el crecimiento económico colombiano respecto a otros departamentos. Desde los noventa, el Valle se ha caracterizado por la ampliación en sus actividades industriales, financieras y agrícolas, y por poseer uno de los mayores ingresos por habitante (PIB per-cápita) en el país (17,7 millones) (Departamento Administrativo Nacional de Estadística [DANE], 2016). No obstante, en la primera década del siglo XXI registró una marcada desaceleración (figura 1), por debajo del promedio nacional.

Este departamento, a diferencia de la economía nacional, presenta menor dependencia de la actividad extractiva petrolera, al basar su industria en subsectores como el de química y el de alimentos y bebidas, con una participación en el PIB departamental de la industria de $23,2 \%$ y $42,0 \%$, respectivamente (DANE, 2016). Por otro lado, la industria del departamento es una gran transformadora de materia prima importada y de bienes de capital; ambos rubros abarcan aproximadamente el $88,0 \%$ de las importaciones totales del departamento (Hernández y Raffo, 2016). En consecuencia, la baja dependencia del sector minero-energético hace que los cambios en los precios del petróleo afecten de manera distinta la actividad económica del departamento.

Los efectos que generan los cambios de los precios del petróleo sobre la economía colombiana han sido un campo de estudio por varios autores; sin embargo, en el orden regional no existe literatura que aborde el efecto de dicha variable sobre los departamentos colombianos con una estructura poco o nada dependiente de la actividad petrolera, como el caso del Valle del Cauca. Algunos estudios sobre la fluctuación de los precios del petróleo y sus efectos en Colombia, desarrollados por autores como Uribe y Ulloa (2011), González y Hernández (2016) y Gómez (2015), en los que analizan los choques registrados en la actividad petrolera sobre la economía colombiana, concluyen que un aumento en los precios internacionales del crudo forja un crecimiento adicional en el índice de empleo, en la demanda agregada y provoca mejoras en otros indicadores macroeconómicos; pero, a su vez, se transmiten presiones inflacionarias. Lo anterior permite inferir que un país exportador de petróleo como Colombia se ve impactado positivamente por las fluctuaciones alcistas en su precio.

La variable precios de petróleo en países exportadores de crudo genera un gran efecto en la tasa de cambio y puede promover una posible enfermedad holandesa: se desarrolla un impacto negativo sobre la industria de un país, debido a que la revaluación de la moneda interna por el gran número de exportaciones del sector mineroenergético desincentiva la producción y estimula la importación, tema ampliamente estudiado por Corden (1984), Égert y Leonard (2008), Poncela, Senra y Sierra (2017), entre otros. Una hipótesis de trabajo podría ser que el Valle del Cauca se vea afectado debido a su estructura económica, ya que una gran entrada de divisas causada por altos precios del petróleo generaría una revaluación de la moneda interna y haría menos competitiva la industria departamental. Esto se suma al deterioro del consumo por el lado de otros ingresos externos, como en las remesas, puesto que el Valle del Cauca recibe el $29 \%$ del total anual (Banco de la República, 2017), lo que le representó un poco más de 1500 millones de dólares en 2017 , equivalentes al $4,7 \%$ del PIB y alrededor del $87 \%$ del total exportado por el departamento en el mismo año.

Por lo anterior, son pertinentes estas preguntas: ¿cuáles son los efectos de los precios del petróleo y la tasa de cambio en la economía del Valle del Cauca, Colombia? y ¿cuánto persiste un choque de los precios del petróleo y, en especial, de la tasa de cambio en la economía vallecaucana? Para dar respuesta a estos dos interrogantes, el presente artículo busca estudiar los efectos ocasionados por los impactos del precio del petróleo y la tasa de cambio sobre la economía vallecaucana, mediante la aplicación de dos modelos: un vector 
de corrección de errores (VECM) y un vector autorregresivo en niveles (VAR). Ambos se usan cuando las variables de estudios están cointegradas, lo cual permite realizar un estudio de la relación de largo y corto plazo (Engle y Granger, 1987; Sim, Stock y Wackson, (1990).

El artículo se desarrolla de la siguiente forma: en la siguiente sección se hace la revisión bibliográfica referente a los efectos del precio del petróleo y la tasa de cambio para Colombia y otras economías. Posteriormente se describe la estructura de la economía del Valle del Cauca y el comportamiento de sus principales sectores productivos. En una sección posterior se presentan los modelos econométricos por utilizar y los datos usados en el estudio, con sus respectivas pruebas. Por último, se plantean los resultados y las reflexiones pertinentes.

\section{MARCO DE REFERENCIA}

\section{Efectos del precio del petróleo, la enfermedad holandesa y la tasa de cambio}

Diversas investigaciones identifican efectos diferenciados de las variaciones en la tasa de cambio y el precio de petróleo sobre distintas economías y el fenómeno de la enfermedad holandesa, según sea la posición del país o la región frente al mercado petrolero. Estudios para países exportadores de crudo como Colombia (Uribe y Ulloa, 2011; González y Hernández, 2016) y Venezuela (Mendoza y Vera, 2010) muestran una reacción favorable a las variaciones positivas de los precios del petróleo. De forma contraria, en economías desarrolladas, como la de Estados Unidos, la subida del precio del petróleo impacta en forma negativa (Hamilton, 2003).

Desde un modelo VAR, Kilian y Vigfusson (2011) encuentran para este último país una asimetría de la respuesta ante las alteraciones de los precios del crudo; los autores aluden que los choques positivos tienen un impacto mucho mayor que los negativos. Lo anterior se reafirma en el estudio de Hamilton (2003), donde se identifica que un alza en los precios del petróleo es una de las causas que puede generar una recesión económica en Estados Unidos, debido a que aumentan los costos de producción (como en el caso de los automóviles). Además, los consumidores experimentan una mayor incertidumbre sobre el futuro por el costo de los combustibles, lo que promueve disminución de la demanda de casas, carros y bienes de inversión. Para la economía estadounidense existen diferentes investigaciones, aparte del estudio de Kilian y Vigfusson (2009), que identificaron que un aumento en el precio del petróleo crea un efecto negativo más contundente en la economía que una baja de este; entre ellos están los de Lee, Ni y Ratti (1995), Davis y Haltiwanger (2001), Bernanke (1983), Hamilton (1983), Pindyck (1991) y Balke, Brown y Yücel (2002).

Por su parte, Mendoza y Vera (2010) estudian los efectos de un choque en los precios del petróleo para la economía venezolana; así, identifican que un incremento de los precios del barril del crudo contribuye al crecimiento de dicha economía, mientras que una baja en los precios del petróleo lo reduce, aunque en una proporción menor. Esto se debe a que el sector petrolero es de gran importancia para Venezuela, ya que de este dependen casi exclusivamente sus exportaciones, sus ingresos fiscales, su gasto público y, por tanto, el PIB. Este estudio, en Venezuela, se realizó siguiendo la metodología de Hamilton (2003) y Mork (1989) para la economía estadounidense; allí evidencian la volatilidad de los precios del crudo y luego analizan los efectos de este sobre la demanda agregada, usando los modelos de regresión lineal MCO y GARCH.

Para el caso de Colombia, Uribe y Ulloa (2011) estimaron un vector autorregresivo estructural con variable exógena (S-VARX) y un vector de corrección del error estructural con variable exógena (S-VECX); encontraron efectos positivos de un alza de los precios del crudo sobre indicadores macroeconómicos como el PIB, la manufactura industrial y el empleo, acompañado de un aumento en el indicador de precios por la dinamización de la 
economía. La mejora de estos indicadores se da, en lo fundamental, por el aumento en las expectativas de ingresos por exportaciones petroleras y, en consecuencia, se genera crecimiento en el consumo, mayor recaudo fiscal por regalías petroleras y se afecta positivamente el gasto público.

Por su parte, González y Hernández (2016) identificaron, con un modelo VAR, una relación positiva entre el aumento de los precios del crudo y el crecimiento del PIB jalonado por el consumo, el alza de las exportaciones por la venta de crudo y la inversión extranjera directa. Gómez (2015), aunque llega una a conclusión parecida, sostiene que los impactos de los precios del petróleo en el ciclo económico se desvanecen rápidamente debido a que la producción y refinación del hidrocarburo participa con menos del 10\% del PIB colombiano.

Uno de los impactos negativos del aumento de los precios del crudo en las economías exportadoras como la colombiana es la llamada enfermedad holandesa, que hace referencia al impacto negativo sobre la industria de un país por la revaluación de la moneda interna, a raíz del gran número de exportaciones del sector minero-energético. Estudios como el de Corden (1984), a través de un modelo analítico, deducen que la enfermedad holandesa genera desindustrialización por la revaluación de la moneda interna, a lo que se suman los precios rígidos acarreados por la entrada de divisas al país. Entre tanto, Égert y Leonard (2008) demuestran empíricamente que el auge de los sectores mineroenergéticos en un país incentiva la entrada de capitales, lo que jalona la revaluación de la moneda interna. Lartey (2008), desde una perspectiva econométrica, concluye que el aumento de la inversión extranjera, aunque no sea causada solamente por el sector minero-energético, genera revaluación de la moneda interna de un país; por ello, el autor encuentra que el auge del sector petrolero crearía desempleo por cuanto este sector no es intensivo en mano de obra.

En línea con lo anterior, Perilla (2010) descubre que una fluctuación en el precio del petróleo provoca efectos asimétricos en la economía colombiana, por cuanto su incremento no impacta significativamente el crecimiento económico, pero una disminución sí lo contrae. En un análisis sectorial, el autor deduce que los impactos de las fluctuaciones del precio del petróleo tienen efectos diferentes: en el caso de la industria y la agricultura, reduce significativamente las exportaciones, debido a que la revaluación del peso colombiano introduce pérdida de competitividad; por otro lado, sectores como el de la construcción se ven favorecidos por la facilidad de la importación de insumos y bienes de capital a menor costo. Lo anterior permite concluir que los efectos del petróleo dependen del sector específico que se trate. Vale resaltar que el modelo econométrico utilizado por este autor es un vector autorregresivo estructural (SVAR).

Igualmente, en otro estudio hecho para Colombia por Poncela, Senra y Sierra (2017) se encuentra evidencia sobre la existencia de cointegración entre, por un lado, los precios de materias primas como el petróleo, el café y el carbón exportados por Colombia y, por otro, la tasa de cambio y la producción manufacturera. Se identifica allí que un aumento de los precios de las materias primas en Colombia genera una apreciación de la moneda; sin embargo, los investigadores no encuentran evidencia de que el tipo de cambio afecte significativamente la industria, o bien, que exista desindustrialización.

Como se ha mencionado, una de las variables que se ve afectada directamente por un aumento del precio del petróleo en los países exportadores es la tasa de cambio. Algunos autores que han estudiado el efecto de esta tasa sobre diversas economías —como Leung y Yuen (2007) para Canadá, y Hua (2007) y Frenkel y Ros (2006) para países latinoamericanos (Argentina, Brasil, Chile y México)— identifican que una apreciación de la moneda nacional genera pérdida de competitividad de los productos nacionales en mercados internacionales, debido al aumento relativo de sus precios.

Con respecto a la trasmisión de los efectos directos que sufre la economía colombiana derivados de la tasa de cambio, Sierra y Manrique (2014) y Peláez y Sierra (2016) evalúan los impactos de la tasa de cambio real sobre el valor agregado y el 
empleo por sectores industriales en Colombia. Los autores señalan que el efecto de la tasa de cambio en los sectores depende de varios factores, como el porcentaje de producción que va a los mercados internacionales y el porcentaje de insumos importados. De igual manera, una apreciación del peso colombiano genera un impacto negativo en el empleo en dieciocho industrias colombianas, y de forma inversa, causa un impacto positivo en siete subsectores industriales. En concreto, Sierra y Manrique (2014) evalúan que una apreciación del peso en un $1 \%$ produce un impacto negativo en $0,26 \%$ en el valor agregado de la industria.

En los estudios regionales, Alonso y Patiño (2007) analizaron series anuales del PIB y las exportaciones entre 1960 y el 2000 para el Valle del Cauca; así, determinaron que el PIB local está cointegrado con las exportaciones. Este resultado adquiere relevancia en esta investigación por cuanto las exportaciones están ligadas al tipo de cambio y a su vez podrían estar relacionados con las variaciones en el precio del petróleo. Los autores desarrollaron una investigación a través de un vector autorregresivo en niveles, lo cual permitió concluir que un choque positivo en el PIB genera un incremento en las exportaciones; sin embargo, no es evidente que un aumento en las exportaciones produzca un crecimiento del PIB. Vale resaltar que las conclusiones de Alonso y Patiño (2007) dejan muchos interrogantes sobre el comportamiento de las exportaciones y la dinámica de la actividad económica del departamento. De acuerdo con North (1990), la importancia de las exportaciones en una economía pequeña como la del Valle del Cauca se da porque estas son la base de su crecimiento económico regional, cuando el mercado interno se torna insuficiente al estar totalmente cubierto por la oferta fabril interna.

Finalmente, se puede identificar que los países con vocación exportadora de crudo pueden estar expuestos a una enfermedad holandesa a causa de la masiva entrada de divisas originada por el sector minero-energético. Igualmente, se puede observar que existe poca literatura sobre el efecto de las variaciones de los precios de las materias primas exportadas desde Colombia - como el petróleo, el café y el carbón- en sus regiones, ya que la mayoría de los análisis se han realizado en forma agregada para la economía del país.

\section{ECONOMÍA DEL VALLE DEL CAUCA}

En esta sección se analiza la economía del Valle del Cauca, abordando la dinámica de la demanda agregada y algunos de sus principales sectores productivos. En algunos casos se utilizó el filtro de Hodrick Prescott, que permite descomponer una serie en ciclo y tendencia, dado que se realiza un proceso de minimización de la varianza del componente del ciclo respecto a la tendencia con un parámetro de suavización $\lambda$. La economía colombiana y departamental cuentan con comportamientos asociados, pues existe una correlación positiva del $82 \%$ de las tasas de crecimiento (coeficiente de correlación de 0,82 ); la tasa de crecimiento económico del departamento ha tenido picos más pronunciados que el país en su conjunto. En la gráfica 1 se puede observar la conducta de la tasa de crecimiento del PIB nacional frente a la departamental en el periodo 2001-2015; se muestra allí que en el periodo 20052010 hubo un crecimiento máximo de la demanda agregada del país de $6,90 \%$, frente a un $8,80 \%$ del Valle del Cauca. En 2009, la tasa de crecimiento del departamento fue de $0,72 \%$, en tanto la colombiana fue de $1,65 \%$; estos fueron los periodos de mayor desaceleración.

Entre 2002 y 2014 el Valle del Cauca tuvo rápidas fluctuaciones en la tasa de crecimiento económico. Durante el periodo 2002-2006 tuvo expansiones mayores al 3\% (excepción en 2005). Entre 2007 y 2009 la economía empezó a desacelerase continuamente hasta llegar a un crecimiento del $0,72 \%$, probablemente por la crisis subprime. Por último, entre 2010 y 2015 la economía departamental empieza a tener una permanente recuperación e incluso llega a expandirse por encima del promedio nacional. 
Gráfica 1.

Historial de la tasa de crecimiento del PIB de Valle del Cauca vs. Colombia, 2001-2015

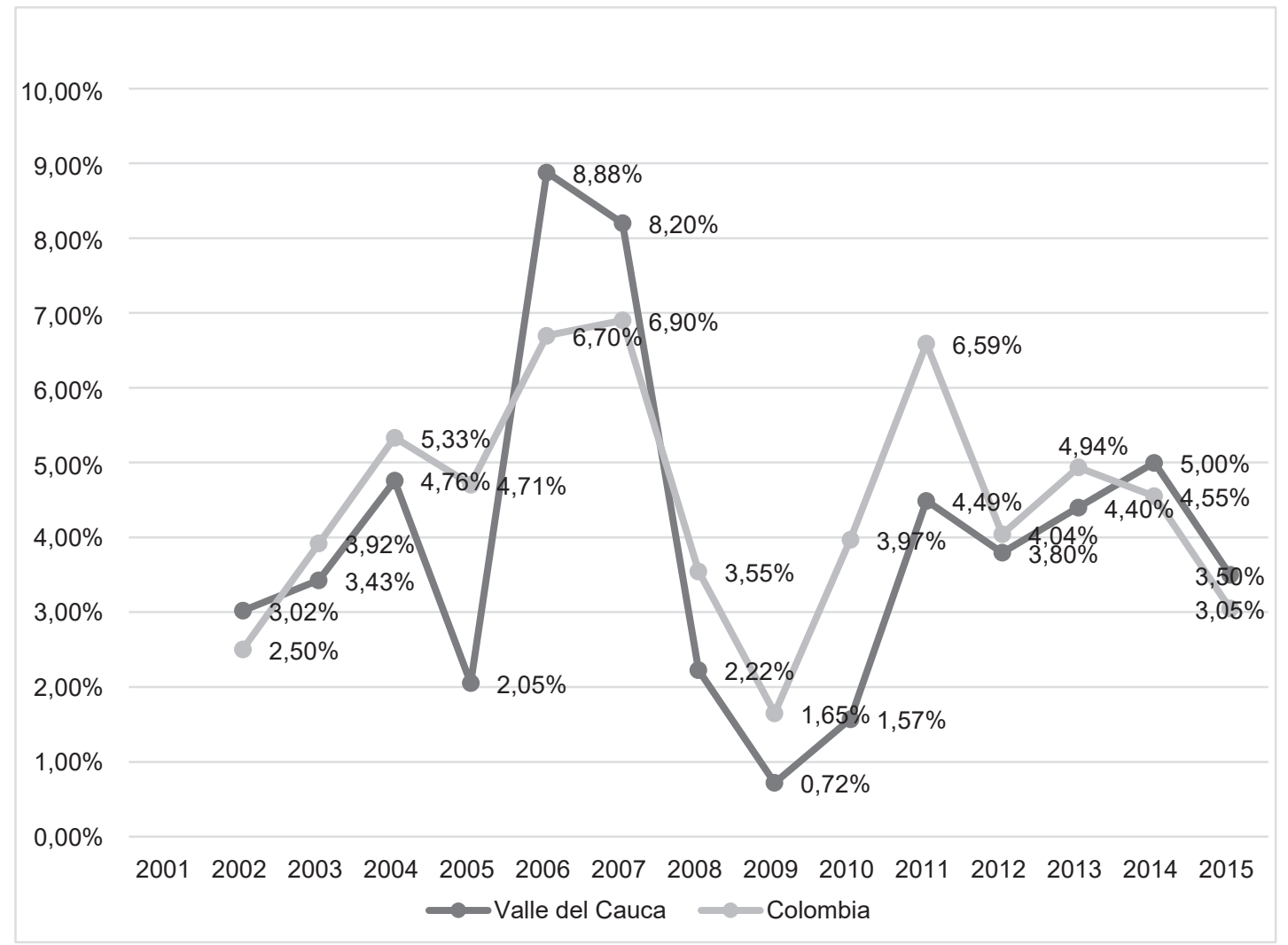

Fuente: elaboración del autor a partir de datos del Banco de la República y el DANE (2016).

Respecto a los precios del petróleo, su relación con el crecimiento de la economía colombiana y departamental se puede observar en la gráfica 2 . Entre 2006 y 2007 el precio del petróleo comenzó a subir de forma considerable en aproximadamente un $50 \%$, lo que impulsó un periodo de aceleración para la economía nacional, mientras que la economía departamental empezó a desacelerase. Entre 2008 y 2013 el precio del petróleo subió aproximadamente un $80 \%$ después de la crisis subprime, y la economía nacional creció por encima de la departamental; pero cuando los precios iniciaron su descenso en 2014, la economía departamental comenzó a acelerarse en contraste con el comportamiento de la nacional. Se hace evidente en este periodo que los auges del sector minero-energético impulsan mayores tasas de crecimiento de la economía colombiana que la economía vallecaucana, y cuando lo precios del petróleo son relativamente bajos, la economía departamental crece a una mayor tasa que la economía nacional.

\section{Caracterización sectorial}

\section{Industria}

La industria vallecaucana está conformada por múltiples subsectores que dividen su contribución en el valor bruto de la producción, como se muestra en la tabla 1. Por otro lado, al observar la gráfica 3 , que describe el índice de producción industrial del Valle 
Gráfica 2.

Precio del petróleo WTI, años vencidos, 2001-2017

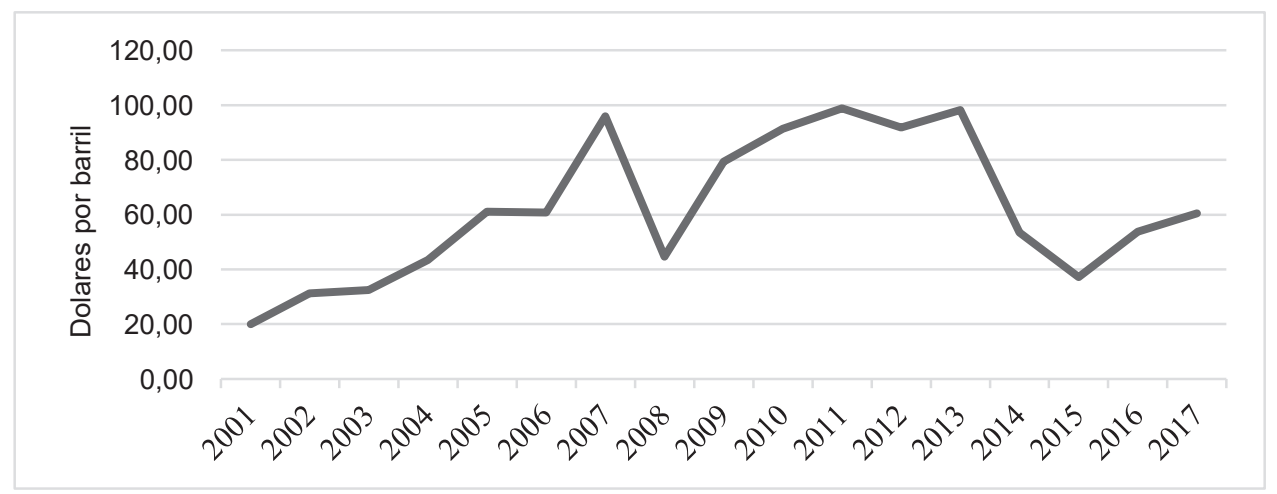

Fuente: elaboración del autor con base en datos de FRED St of. Louis

del Cauca (IPIR) —indicador implementado por el Banco de la República de Cali-, se infiere una dinámica beneficiosa para todo el sector industrial de la región. Como se evidencia a partir de 2005, se presentó un cambio de nivel producido por la apertura de las primeras plantas de etanol, lo que generó un choque positivo en todo el sector productor de la región. Este impulso recibido por la industria local implicó un cambio estructural en el nivel de largo plazo; dicho impacto en la producción de biocombustibles produjo un leve incremento en la tendencia de largo plazo para el sector manufacturero.

Tabla 1.

Participación de subsectores industriales, 2016

\begin{tabular}{|c|c|}
\hline Subsector industrial & Participación (\%) \\
\hline Alimento y bebidas & 40 \\
\hline Químicos & 15 \\
\hline Metalúrgicos & 12 \\
\hline Combustibles & 8 \\
\hline Papel & 7 \\
\hline Textiles, confecciones, cuero y \\
calzado & 4 \\
\hline Eléctricos & 3 \\
\hline Vidrios y minerales & 3 \\
\hline Cauchos y plásticos & 3 \\
\hline Vehículos y sus partes & 1 \\
\hline Maquinaria y equipo & 1 \\
\hline Madera & 0 \\
\hline Resto de la industria & 2 \\
\hline Total & 100 \\
\hline
\end{tabular}

Fuente: EAM (2016).
Como se puede observar en la gráfica 4 , en octubre de 2006 la tendencia del IPIR tuvo el mayor crecimiento: un $6,6 \%$ con respecto al mismo mes del año anterior. En diciembre de 2008 su tendencia alcanzó el menor crecimiento: 0,3\%; en octubre de 2011 se presentó otro pico de crecimiento importante: $1,7 \%$, y en septiembre de 2016 la tasa anual de crecimiento real fue de $1,8 \%$.

En lo referente al comercio internacional, las exportaciones del sector industrial del Valle del Cauca desempeñan un papel importante en la economía local, al representar aproximadamente el $97,0 \%$ de todos los despachos al exterior, con destino a Estados Unidos, Ecuador y Perú, con una participación de $20,2 \%, 18,1 \%$ y 13,8 \%, respectivamente (Cámara de Comercio de Cali, 2017). Hernandez y Raffo (2016) describen que subsectores como el de papel y cartón, otros alimentos, azucarero y químicos son trascendentales para el sector externo, con aportes en su orden de $17,5 \%$, $15,1 \%, 11,2 \%$ y $11 \%$, entre 2010 y 2014 . La clase industrial maquinaria y equipo le siguió en importancia con $9,4 \%$, y la de caucho y plástico con $8,2 \%$.

Por otra parte, las importaciones del sector industrial del Valle del Cauca provienen básicamente de China y Estados unidos; para 2014 la suma de estas fue aproximadamente del $30 \%$ del total. Estados Unidos ha venido perdiendo participación al pasar los años, y China ha presentado un 
Gráfica 3.

IPIR, ciclo y tendencia, 2000-2016

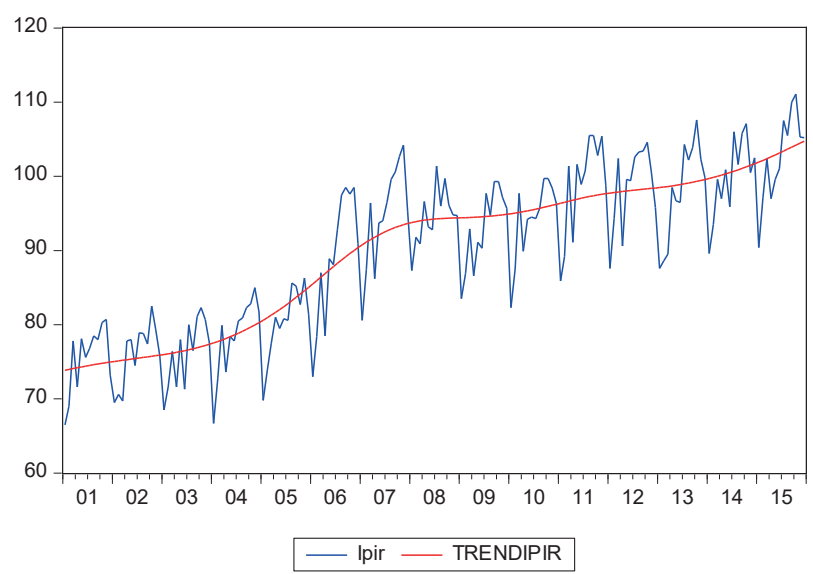

Fuente: elaboración del autor con base en datos del Banco de la República (2016) y Hodrick-Prescott Filter, con una lambda 14400.

Gráfica 4.

Variación de tasa de crecimiento, tendencia interanual del IPIR, 2000-2016

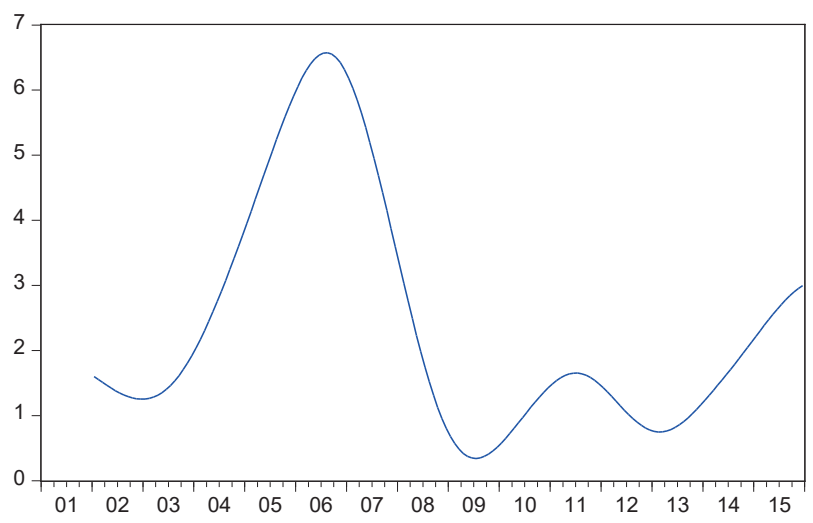

Fuente: elaboración del autor con base en datos del Banco de la República (2016) y Hodrick-Prescott Filter, con una lambda 14400.

comportamiento inverso. Hernández y Raffo (2016) mencionan los tipos de bienes que se importan en el Valle del Cauca; así, es posible inferir que el $81 \%$ de las importaciones entre 2010 y 2014 fueron productos intermedios y de capital; esto a su vez muestra indicios de que la producción del departamento depende de sus importaciones (Hernández y Raffo, 2016), lo cual está directamente ligado a la tasa de cambio.

\section{Agricultura}

La agricultura en el departamento se concentra básicamente en el cultivo de caña de azúcar, destinado a diferentes fines como la producción de alcohol carburante, etanol, panela, miel, ácido cítrico, nitrato de sodio deshidratado, citrato de calcio, acetato de etilo, vinagre y abonos. Este clúster azucarero es de vital importancia para el país, ya que en los noventa representaba aproximadamente el $10 \%$ de la producción agrícola colombiana y a su vez generaba más del $8 \%$ de empleo en la industria alimentaria. Este clúster está ubicado en el valle geográfico del río Cauca, que involucra a los departamentos de Cauca y Valle del Cauca. Estos departamentos albergan el $98 \%$ de la caña sembrada para la producción azucarera; el Valle alberga el $80 \%$ del sembrado, y el Cauca, el $18 \%$ restante. Una de las razones más importantes por las que existe esta aglomeración de producción azucarera es la cercanía al puerto de Buenaventura, lo cual reduce los costos de transporte (Martínez, 2006). Se debe resaltar que el clúster azucarero es proveedor de grandes industrias colombianas como bebidas, confites y alimentos. En 2007 los efectos indirectos de los ingenios azucareros sobre la economía colombiana fueron de 2,9 billones de pesos (Arbeláez, Estacio y Olivera 2010)

Para estudiar el comportamiento de este sector, se analizaron las toneladas de molienda de caña en el Valle del Cauca. La tendencia de la molienda de caña (gráfica 5) ha tenido en promedio tasas de crecimiento positivas desde el 2000. Sus picos de crecimientos más altos respecto al mismo mes del año inmediatamente anterior se ven en junio de 2002 y septiembre de 2013, cuando su tasa de crecimiento fue de $5,6 \%$ y $5,8 \%$, respectivamente. La tasa de crecimiento más baja se vio en enero del 2007 , con un $-2,0 \%$ (gráfica 6 ). 
Gráfica 5.

Ciclo y tendencia de la molienda de caña de azúcar

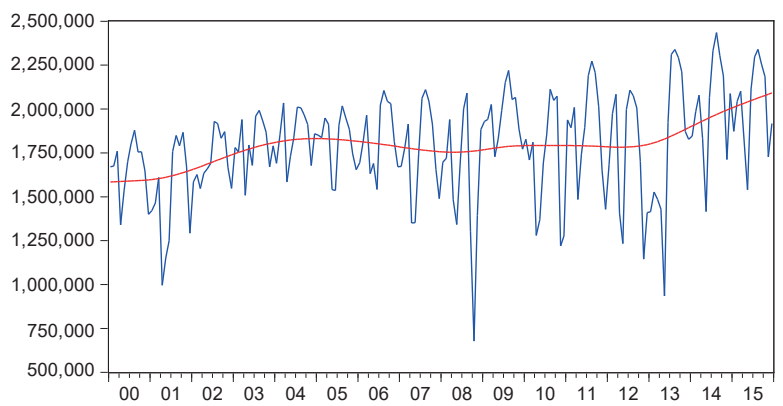

— Molienda de caña — TREND

Fuente: elaboración del autor con base en datos del Banco de la República (2016) y Hodrick-Prescott Filter, con una lambda 14400.

Gráfica 6.

Variación porcentual de la tendencia de molienda de caña de azúcar interanual

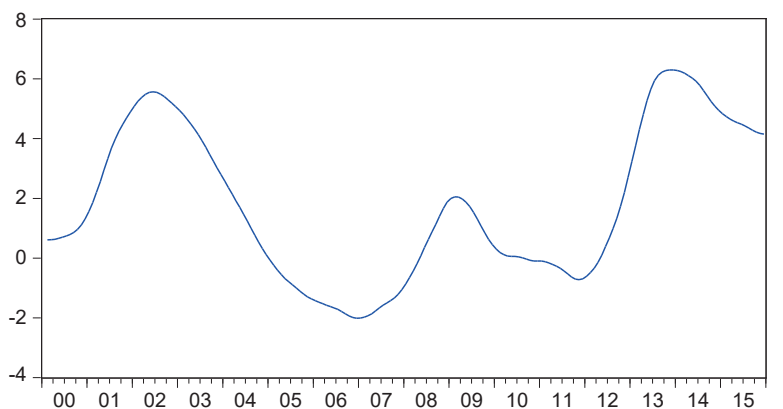

Fuente: elaboración del autor con base en datos del Banco de la República (2016) y Hodrick-Prescott Filter, con una lambda 14400.

\section{Sector pecuario}

En el subsector de alimentos, el departamento tiene clústeres importantes como la producción de proteína blanca y azúcar. La producción de proteína blanca y sus derivados es la segunda a nivel nacional (Cámara de Comercio de Cali, 2016), lo que los hace parte de la industria del departamento. La gráfica 7 muestra la producción de carne de pollo en toneladas y su tendencia, lo que permite analizar dicho subsector a partir de sus históricos. Así, el estudio arrojó que la producción de carne de pollo ha tenido un aumento paulatino entre 2005 y 2015, con cambios estructurales en su tendencia en 2011. La gráfica 8 muestra la variación anual de la tendencia de la producción de carne de pollo, que ha tenido variaciones positivas, aunque ello ha estado acompañado de desaceleración en el periodo 2004-2010. Igualmente, el mayor punto de crecimiento fue de $18,2 \%$ en septiembre del 2013 y un punto mínimo de $0,8 \%$ en junio del 2010.

Gráfica 7.

Producción de carne de pollo y su tendencia

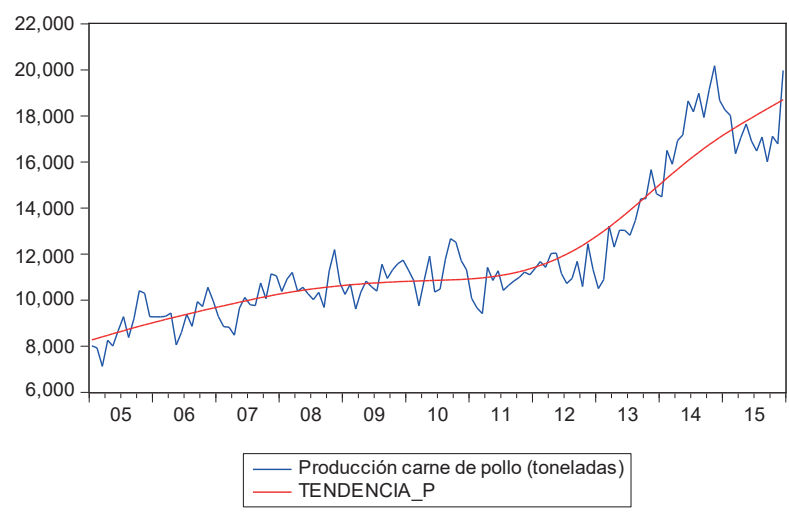

Fuente: elaboración del autor con base en datos del Banco de la República (2016) y Hodrick-Prescott Filter, con una lambda 14400.

Gráfica 8.

Variación porcentual de la tendencia de producción de carne de pollo interaunal

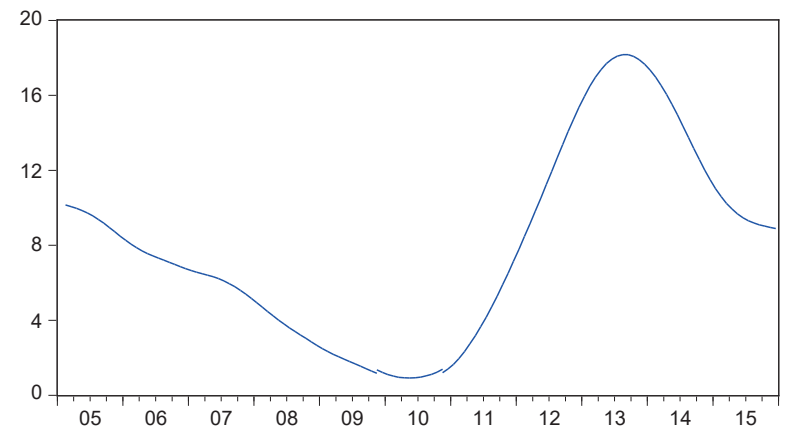

Fuente: elaboración del autor con base en datos del Banco de la República (2016) y Hodrick-Prescott Filter, con una lambda 14400.

La gráfica 9 muestra la producción de huevos por toneladas en el Valle del Cauca, la cual tuvo un crecimiento considerable desde 2005. Por su parte, en la figura 10 se observa la variación 
anual de la tendencia; vale señalar que en octubre de 2007 el crecimiento llegó a niveles de -3\% (el punto mínimo), y en los años posteriores hubo una recuperación, con un punto máximo de $11,03 \%$ en septiembre de 2015. Este análisis se hace a partir de la producción de huevos desde 2004 hasta 2015.

Gráfica 9.

Producción de huevos y su tendencia

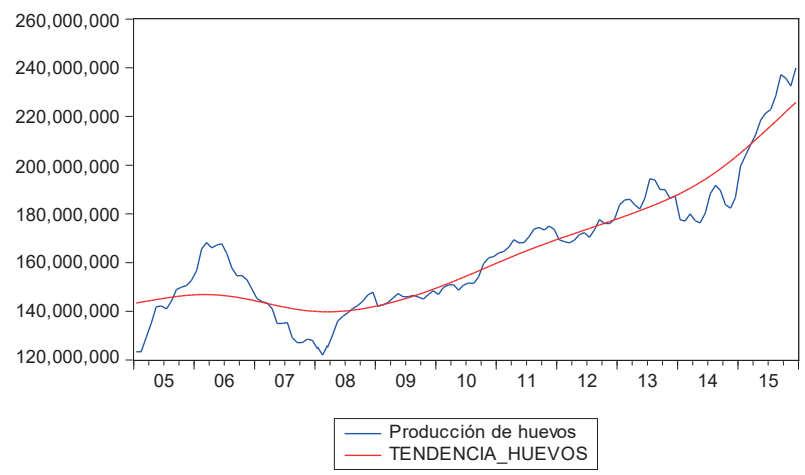

Fuente: elaboración del autor con base en datos del Banco de la República (2016) y Hodrick-Prescott Filter, con una lambda 14400.

Gráfica 10.

Variación porcentual de la tendencia de producción de huevo año a año

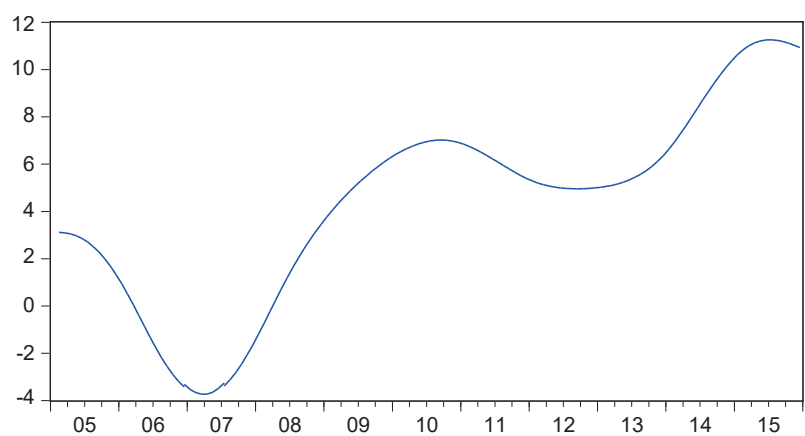

Fuente: elaboración del autor con base en datos del Banco de la República (2016) y Hodrick-Prescott Filter, con una lambda 14400.

\section{Sector construcción}

En la gráfica 11 se puede ver que la venta de vivienda en Cali y sus alrededores ha tenido fluctuaciones considerables en los últimos años; sus niveles más bajos se dieron entre 2008 y 2009 debido a la crisis inmobiliaria de 2008. La gráfica 12 muestra que en los primeros meses de 2008 la tendencia de la venta de vivienda tuvo un decrecimiento del $12,8 \%$, recuperándose en septiembre de 2009 y repuntando a finales de 2010 con tasas de crecimiento de $9,1 \%$. Este comportamiento mostró una recuperación rápida del sector. Los mejores momentos del sector se han visto en abril del 2014, con tasas alcistas de 15,4\%. En 2015 y 2016 se han visto tasas de crecimiento entre $14,5 \%$ y $15 \%$.

Gráfica 11

Venta de vivienda nueva en Cali y municipios aledaños, y su tendencia

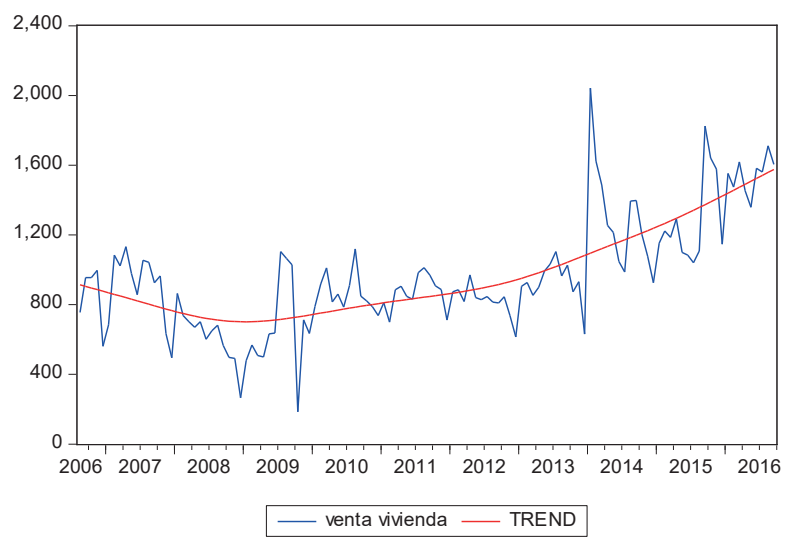

Fuente: elaboración del autor con base en datos del Banco de la República (2016) y Hodrick-Prescott Filter, con una lambda 14400.

Gráfica 12 .

Variación de crecimiento porcentual de la tendencial interanual de ventas de viviendas de Cali, Jamundí y Yumbo

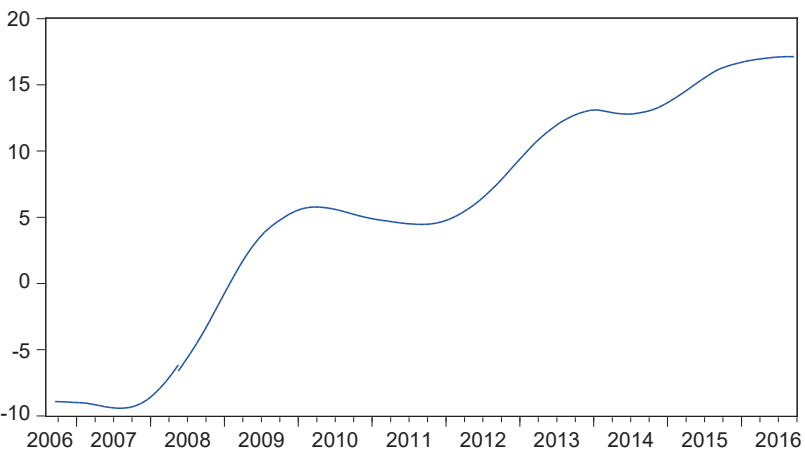

Fuente: elaboración del autor con base en datos del Banco de la República (2016) y Hodrick-Prescott Filter, con una lambda 14400. 


\section{Sector transporte}

Paran analizar el sector de transporte del Valle del Cauca, se usó como proxy el tráfico de vehículos de particulares por los 10 principales peajes del departamento, e igualmente se utilizó la misma variable para el transporte de carga. El tráfico por las principales vías del departamento ha aumentado extensamente desde 2003, tanto en el paso de vehículos particulares como de carga (gráficas 13 y 14). El crecimiento de la tendencia de tráfico de vehículos ha tenido sus picos a finales del 2014 e inicios del 2015, con tasas alcistas tendenciales de hasta $6,50 \%$ comparadas con el año inmediatamente anterior para carros particulares. Igualmente, la tasa de aumento de la tendencia de esta variable ha sido mínimo de 5,0\% (gráfica 15). Para el tráfico de carga, los datos se comportan de una manera muy similar; su pico de crecimiento tendencial fue en los últimos meses de 2014, con tasas de 4,4\%, y su crecimiento mínimo no ha sido menor al $3 \%$.

Gráfica 13.

Tráfico de vehículos particulares en diez peajes del departamento y tendencia interanual

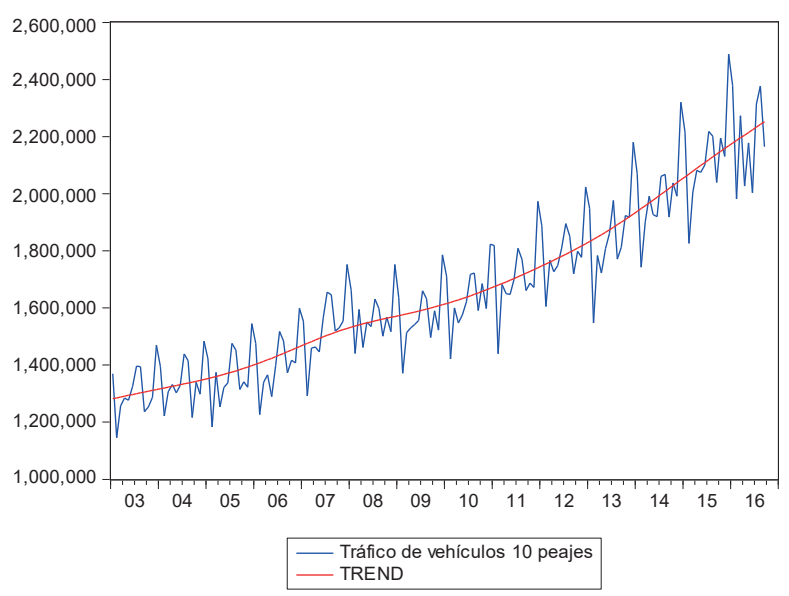

Fuente: elaboración del autor con base en datos del Banco de la República (2016) y Hodrick-Prescott Filter, con una lambda 14400.
Gráfica 14.

Tráfico de vehículos de carga en diez peajes del departamento $y$ tendencia interanual

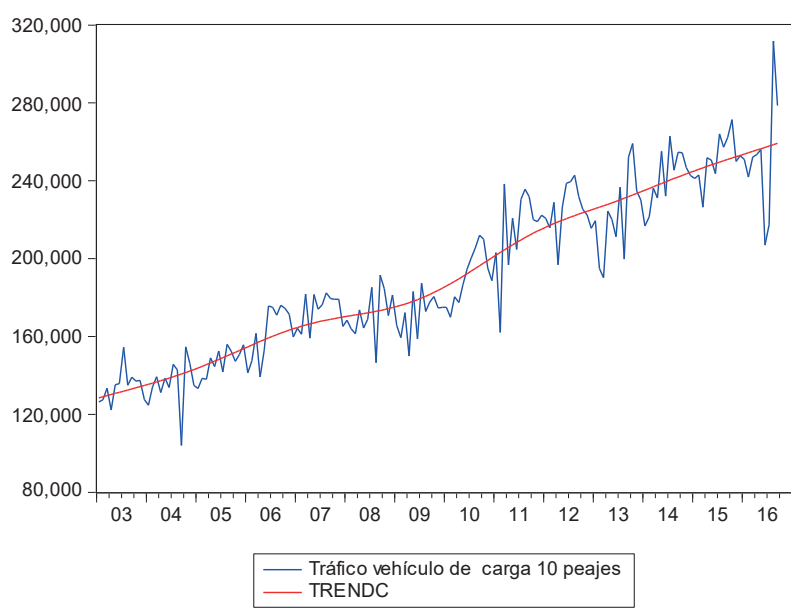

Fuente: elaboración del autor con base en datos del Banco de la República (2016) y Hodrick-Prescott Filter, con una lambda 14400 .

Gráfica 15.

Variación de crecimiento porcentual interanual tendencial en transporte de carga y tráfico de vehículos particulares

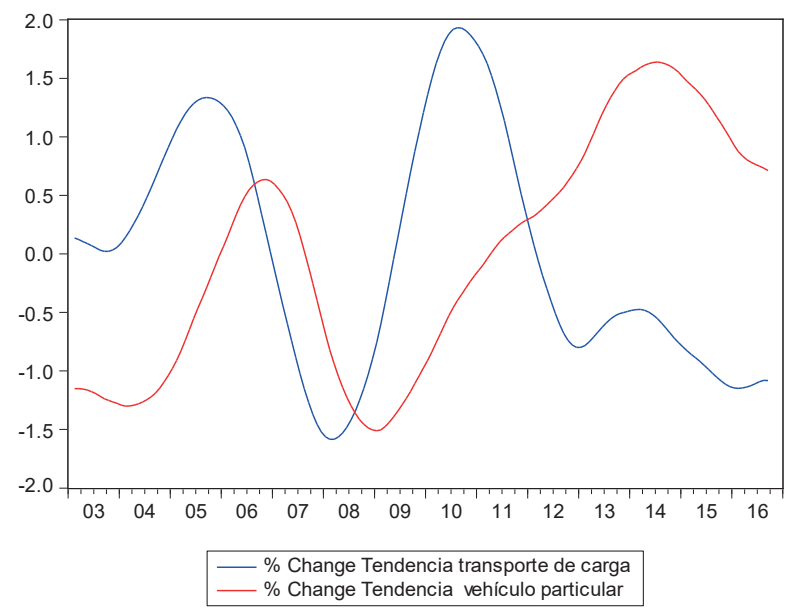

Fuente: elaboración del autor con base en datos del Banco de la República (2016) y Hodrick-Prescott Filter, con una lambda 14400. 


\section{Sector externo}

Resulta de interés comprender que la relación entre las exportaciones e importaciones en el Valle del Cauca están correlacionadas en el tiempo, por cuanto desde la apertura económica a comienzos de los noventa se importa materia prima para ser transformada en la región y posteriormente ser exportada a los mercados de Centroamérica, el Caribe y Suramérica. Esta situación se da debido a que muchas multinacionales tienen establecidas en la región plantas con alta inversión para suplir mercados más pequeños. El Valle del Cauca ha tenido un déficit comercial constante, donde las exportaciones departamentales son menores que sus importaciones, como se puede observar en la gráfica 16. Igualmente, los ciclos de estas variables tienen comportamientos similares, ya que las exportaciones se deben mayormente a la industria, y esta, por lo general, tiene un tipo de blindaje a una apreciación de la moneda local, debido a que algunas de las materias primas y activos para la producción de dicha actividad económica son importadas y el producto final es por lo general exportado.

Gráfica 16.

Exportaciones e importaciones del Valle del Cauca, 20002015

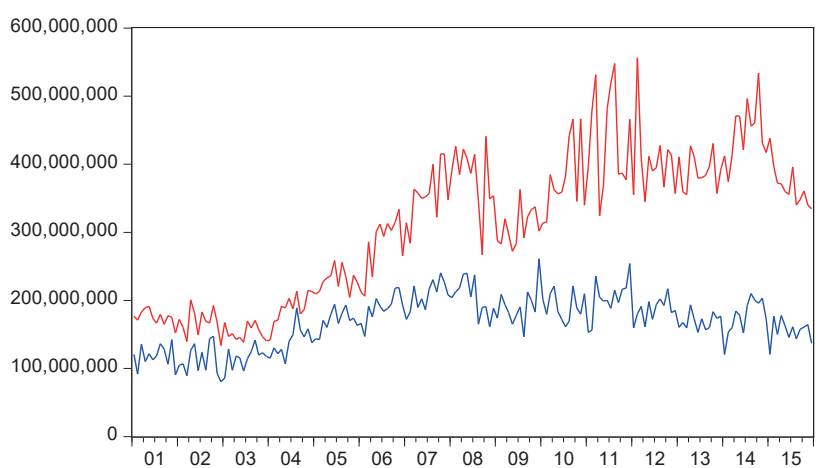

$$
\text { - Exportaciones _- Importaciones }
$$

Fuente: elaboración del autor con base en datos del BR de Colombia (2016).

En síntesis, el Valle del Cauca tiene una economía altamente diversificada que le ha permitido paliar diferentes crisis económicas, dado que algunas de las materias primas y activos para la producción de la actividad industrial son importadas y el producto final es por lo general exportado. En consecuencia, el departamento se ve afectado directamente por la tasa de cambio e indirectamente por las fluctuaciones del precio del petróleo.

\section{METODOLOGÍA EMPÍRICA}

En esta sección se desarrollan dos modelos: un vector de corrección de errores (VECM) y un vector autorregresivo (VAR) en niveles, utilizados para resolver las preguntas de investigación, a saber: ¿cuáles son los efectos de los precios del crudo y la tasa de cambio en la economía del Valle del Cauca en el largo plazo? y ¿cuánto persiste un impacto de los precios del crudo y la tasa de cambio en la economía vallecaucana? También se explican los datos por utilizar, y por último se realizan las pruebas respectivas.

\section{El modelo}

El modelo hace referencia a los efectos que generan los términos de intercambio en economías pequeñas y abiertas, un tema ampliamente estudiando por diferentes autores. Un ejemplo de ello es el estudio de Tokarick (1995) sobre modelos de equilibrio general, donde analiza los efectos de los términos de intercambio, considerando el sector exportador, importador y no transable, al tiempo que plantea que los términos de intercambio son exógenos a la economía. Igualmente, Kose y Riezman (1999) analizan los efectos de los impactos internacionales y contemplan los términos de intercambio como variable de comercio exterior en una economía africana abierta y pequeña. Por otro lado, Jin (2002) investiga el crecimiento de economías regionales en algunas provincias coreanas, mediante el análisis de la relación entre el crecimiento económico nacional y regional a partir de un modelo de economía abierta y pequeña. Este modelo plantea que el crecimiento de las economías regionales está relacionado con el de la economía nacional y la variación de los términos de intercambio. 
En el caso de la economía del Valle del Cauca, esta se ve permeada por los términos de intercambio nacionales dado que el precio del petróleo tiene un gran efecto sobre ellos, aunque la economía departamental no sea exportadora de crudo; en consecuencia, se generan efectos contraproducentes por la apreciación de la moneda interna. El mecanismo de transmisión referido a cómo un impacto positivo en los términos de intercambio afecta a cada una de las variables del agregado nacional se explica en los siguientes párrafos.

Dada la relación que presentan los términos de intercambio con los distintos agregados económicos colombianos, y como consecuencia de su exogeneidad, un choque positivo en los $\mathrm{TI}$, debido a un incremento en los precios de los productos exportados (materias primas), motiva una entrada de divisas extranjeras al país, que incentiva la solicitud de créditos, sobre todo en el sector beneficiado por el apogeo, como el de materias primas; por consiguiente, se favorece la inversión. El mayor ingreso del país ocasiona incrementos sobre todos los sectores productores (directos e indirectos de materias primas), sobre el consumo y, como lo indica Hernández (2013), sobre las utilidades del sector financiero y el gobierno (mayores ingresos fiscales).

La moneda local se aprecia en términos reales en primer lugar debido a la apreciación nominal, ocasionada por la entrada de divisas, y en segundo lugar por el incremento en los precios, producido por el aumento de la demanda en general. Un efecto indirecto, negativo y muchas veces olvidado en el análisis de los impactos de los $\mathrm{TI}$, y que potencialmente afectaría al Valle del Cauca, es el que puede causar la apreciación real de la moneda en la competitividad del sector exportador y el aumento de las importaciones por el detrimento de competitividad de la industria. Ello produce una disminución en el valor agregado y en el empleo de dichos sectores (Sierra y Manrique, 2014; Peláez y Sierra, 2016) $y$, en consecuencia, se impacta directamente a la economía vallecaucana.

Por lo anterior, dado que la economía de este departamento se puede concebir como pequeña y abierta, pero de carácter regional, se estudian algunas variables propuestas por Jin (2002); por ende, se seleccionan las siguientes: las exportaciones e importaciones como variables relacionadas con el comercio exterior, la demanda agregada del Valle de Cauca (PIB Valle) como medidor de actividad económica y una variable que relacione la economía regional con la nacional, la cual es el índice de seguimiento económico para Colombia (ISE). Igualmente, Bjørnland (2009) menciona que al estudiarse una economía abierta se debe incluir una variable como la tasa de cambio, por lo cual se añade el índice de tasa de cambio real para Colombia (ITCR); el precio del barril de petróleo (WTI) como proxy de los términos de intercambio para Colombia, y una proxy de la tasa de interés mundial como variable totalmente exógena, la cual es LIBOR (London Interbank Offered Rate).

\section{Modelo econométrico}

Se utilizan los modelos VECM y VAR en niveles ya que las variables de estudio pueden tener relaciones de equilibro a largo plazo, dado que en un sistema de ecuaciones pueden existir combinaciones lineales que generen vectores de cointegración. El término cointegración, que fue estudiado por Engel y Granger (1987) y Johansen (1991), hace referencia a cuando las variables de estudio producen una relación de equilibrio a largo plazo; por lo tanto, la desviación del equilibrio entre las variables afecta en el corto plazo, al tiempo que las combinaciones lineales de variables no estacionarias y cointegradas generan residuales estacionarios.

Los modelos VAR desarrollados por Sims (1980) permiten resolver el problema de endogeneidad de las variables; para ello, se requiere escribir el sistema de ecuaciones a una expresión reducida y matricial, donde se puede apreciar cómo los choques de una variable afectan tanto a esta como a las demás. Se parte de las siguientes ecuaciones para ejemplificar un VAR de primer orden, donde $u_{t}$ y $Z_{t}$ son I(1), y los errores $\varepsilon_{u t}$ y $\varepsilon_{z t}$ son choques, representados por un proceso ruido blanco con media cero y varianzas $\sigma_{\varepsilon u t}^{2} \sigma_{\varepsilon z \mathrm{t}}^{2}$ constantes. El modelo se escribe como el siguiente sistema de ecuaciones: 
$u_{t}=a_{11}+a_{12} z_{t}+b_{11} u_{t-1}+b_{12} z_{t-1}+\varepsilon_{u t}[]$

$z_{t}=a_{21}+a_{22} u_{t}+b_{21} u_{t-1}+b_{22} z_{t-1}+\varepsilon_{z t}$

El resultado es un modelo de ecuaciones simultáneas con la particularidad de que sus variables son endógenas. De forma matricial, el modelo VAR se escribe de este modo:

$\left[\begin{array}{cc}1 & a_{12} \\ a_{22} & 1\end{array}\right]\left[\begin{array}{l}u_{t} \\ z_{t}\end{array}\right]=\left[\begin{array}{l}a_{11} \\ a_{21}\end{array}\right]+\left[\begin{array}{ll}b_{11} & b_{12} \\ b_{21} & b_{22}\end{array}\right]\left[\begin{array}{l}x_{t-1} \\ z_{t-1}\end{array}\right]+\left[\begin{array}{l}\varepsilon_{u_{t}} \\ \varepsilon_{z_{t}}\end{array}\right]$

$\alpha Y_{t}=\Gamma_{0}+\Gamma_{1} Y_{t-1}+\varepsilon_{t}$

$Y_{t}=B_{0}+B_{1} y_{t-1}+\varepsilon_{t}$

En esta investigación se estima un VECM que puede medir las relaciones de corto y largo plazo. Dado lo anterior, se debe determinar el número de relaciones de cointegración; por lo tanto, se plantea un vector de corrección de errores, el cual, según Pesaran, Shin y Smith (2000), parte del modelo VAR con variables no estacionarias, que en este caso son de orden I(1), lo que se representa de la siguiente manera:

$\mathrm{y}_{\mathrm{t}=} \mathrm{A}_{0}+\sum_{\mathrm{p}=1}^{\mathrm{t}-1} \mathrm{~A}_{\mathrm{p}} \mathrm{y}_{\mathrm{t}-\mathrm{p}}+\mathrm{e}_{\mathrm{t}}$,

Donde $t=1,2,3 \ldots \ldots N$

Diferenciando en los dos lados de la ecuación $\mathrm{Y}_{\mathrm{t}-1}$, pueden reorganizarse los términos; por lo tanto, un modelo VAR se puede representar como un modelo VECM de la siguiente forma:

$\Delta \mathrm{y}_{\mathrm{t}=}=\mathrm{A}_{0}+\Pi \mathrm{y}_{\mathrm{t}-1}+\sum_{\mathrm{p}=1}^{\mathrm{t}-1} \Gamma_{\mathrm{p}} \Delta \mathrm{y}_{\mathrm{t}-\mathrm{p}}+\mathrm{u}_{\mathrm{t}}$

Donde $\Pi=-\left(I_{k}-A_{1}-\ldots-A_{p}\right)$ y $\Gamma_{p}=\left(A_{i+1}+\ldots\right.$ $\left.+A_{p}\right), i=1+2+3 \ldots \ldots p-1 ; y_{t} y$ es un vector de variables no estacionarias $\mathrm{k} \times 1$, П es una matriz singular con tamaño $\mathrm{m} X \mathrm{~m}$, la cual captura las $r$ relaciones de cointegración que estima las relaciones a largo plazo; para ello se debe tener en cuenta que las matrices con un rango $r$ con un tamaño $\mathrm{m} \mathrm{X}$ son de rango completo, y en este caso se puede obtener $\pi=\alpha \beta$ '. La sumatoria de $\Gamma_{p}$ recoge todas las relaciones a corto plazo de cada una de las ecuaciones, $A_{0}$ recoge el vector de constates del sistema (Ricci y Ramojo, 2012).

Se plantea un modelo de ecuaciones simultáneas conformado por el logaritmo de PIB del Valle del Cauca, el logaritmo de sus exportaciones, el logaritmo de sus importaciones, el índice de seguimiento económico para Colombia (ISE), el logaritmo de los precios del petróleo WTI y el índice de tasa de cambio real (ITCR). Según Bjørnland (2009), en este tipo de modelos se debe establecer un orden de exogeneidad de las variables, donde la primera no reacciona a las fluctuaciones de las otras debido a su relación contemporánea, lo cual no permite analizar cuáles se verán más afectadas por los choques de las otras. Dado lo anterior, $Y_{t}$ es un vector $7 \times 1$ de variables endógenas organizadas por su orden de exogeneidad:

$Y_{t}=[$ Tim, WTI, ITCR, ISE, Expo, Impo, PIBvalle]' [7]

\section{Datos}

Los datos disponibles para este estudio provienen de una base de datos recopilada por el Banco de la República, Cali, así como del DANE y la base de datos de FRED St. Luis. Para ello se tuvieron en cuenta variables representativas de la actividad económica nacional y departamental, entre las que se encuentran el indicador de seguimiento económico para Colombia (ISE) construido por el DANE, el logaritmo del PIB del Valle del Cauca y el logaritmo de las exportaciones e importaciones del departamento. Como variables externas se utilizan el logaritmo del precio del barril del crudo (WTI), el índice de tasa de cambio real (ITCR) ${ }^{1}$ y una proxy de la tasa de interés mundial LIBOR (London Interbank Offered Rate). Se debe tener en cuenta que la tasa de cambio real está dada por la siguiente expresión:

1 Todas las series expresadas en moneda corriente fueron deflactadas con el indicador de producción industrial de Estados Unidos (Industrial Production Index, Index EEUU $2012=100$, Monthly, Seasonally Adjusted). 
ITCR $=S \times \frac{p}{p_{f}}$

Donde ITCR es el precio relativo de los bienes nacionales frente a los extranjeros, que está ajustado a la tasa de cambio nominal $(S)$, los precios nacionales $(p)$ y los precios internacionales (Pf). Por último, se tomaron datos mensuales desde enero de 2001 hasta diciembre de 2015.

\section{Nivel de integración de las series}

En principio, para realizar un vector de corrección de error con variables externas o un vector autorregresivo en niveles, las variables de estudio deben cumplir con la relación de cointegración. Se dice que las series en niveles están cointegradas cuando su combinación lineal genera residuales estacionarios. Esto indica que el comportamiento de las series presenta patrones de conducta relacionados que impiden su libre movimiento, porque su dinámica está enlazada en el largo plazo, donde converge a un estado de equilibrio (Engle y Granger, 1987). Para identificar el nivel de integración de las series, se realizan pruebas de raíz unitarias "Augmented
Dickey-Fuller test static y Phillip-Perron test statistic"; la hipótesis nula de estas pruebas plantea la existencia de raíces unitarias, lo que quiere decir que las variables son integradas de orden uno, por lo que no son estacionarias.

La tabla 2 muestra las siete (7) series en niveles por usar en el modelo; como es de esperarse, todas presentan una raíz unitaria a cualquier nivel de significancia, por lo que se prosigue a realizar la prueba con la primera diferencia de las series. Se evidencia, entonces, que todas las variables en su primera diferencia se transforman en variables estacionarias, lo que permite concluir que las variables son integradas de orden I(1).

\section{Cointegración}

Para determinar el número de relaciones de cointegración en la matriz $\Pi$, dado que la existencia de estas son necesarias para una correcta estimación de los modelos VECM o VAR en niveles, se realiza la prueba desarrollada por Johansen $(1991,1995)$, donde se plantea la hipótesis nula de la existencia de (r) relaciones de cointegración, en contraste de $(\mathrm{k})$ relaciones de congregación, donde $(\mathrm{k})$ muestra el número de variables endógenas exponiendo un

Tabla 2.

Pruebas de raíces unitarias para las variables de estudio

\begin{tabular}{|c|c|c|c|c|c|c|c|c|}
\hline \multirow{4}{*}{ Variables } & \multicolumn{4}{|c|}{ Augmented Dickey-Fuller test static } & \multicolumn{4}{|c|}{ Phillip-Perron test statistic } \\
\hline & \multicolumn{2}{|c|}{ ADF in levels } & \multicolumn{2}{|c|}{$\begin{array}{l}\text { ADF in first } \\
\text { differences }\end{array}$} & \multicolumn{2}{|c|}{ PP in levels } & \multicolumn{2}{|c|}{$\begin{array}{l}\text { PP in first } \\
\text { differences }\end{array}$} \\
\hline & t-Statistics & Prob. & t-Statistics & Prob. & t-Statistics & Prob. & t-Statistics & Prob. \\
\hline & \multicolumn{2}{|c|}{ Trend and Intercept } & \multicolumn{2}{|c|}{ None } & \multicolumn{2}{|c|}{ Trend and Intercept } & \multicolumn{2}{|c|}{ None } \\
\hline PIB Valle del Cauca & $-2,019$ & 0,5867 & $-10,520$ & 0,0000 & $-1,81$ & 0,6960 & $-17,184$ & 0,0001 \\
\hline ITCR_ & $-2,057$ & 0,5663 & $-10,228$ & 0,0000 & $-1,03$ & 0,9373 & $-5,451$ & 0,0000 \\
\hline Exportaciones del Valle del Cauca & $-2,406$ & 0,3752386 & $-11,227$ & 0,0000 & $-0,41$ & 0,5328 & $-37,184$ & 0,0000 \\
\hline Importaciones del Valle del Cauca & 0,310 & 0,7743 & $-10,724$ & 0,0000 & $-0,39$ & 0,5416 & $-32,853$ & 0,0000 \\
\hline $\begin{array}{l}\text { Indicador de seguimiento económico } \\
\text { (ISE) }\end{array}$ & $-2,732$ & 0,2250 & $-18,617$ & 0,0000 & $-3,18$ & 0,0925 & $-19,022$ & 0,0000 \\
\hline Precio petróleo WTI construida (IPP) & $-2,021$ & 0,5855 & $-9,058$ & 0,0000 & $-1,80$ & 0,7030 & $-9,02$ & 0,0000 \\
\hline Tim & $-1,825$ & 0,3675 & $-9,649$ & 0,0000 & $-2,08$ & 0,2521 & 10,022 & 0,0000 \\
\hline
\end{tabular}

Nota: la hipótesis nula de esta prueba es de existencia de raíz unitaria.

Fuente: elaboración del autor con base en datos del BR de Colombia (2016) y DANE (2017). 
modelo VAR con variables de orden I(1), que es un modelo con residuales estacionarios. Las anteriores hipótesis se contrastan con el estadístico de la traza determinado por la siguiente ecuación:

$\lambda_{\text {traza }(r)}=-T \sum_{i=r+1}^{p} L N\left(1-\lambda_{i}\right)$

Donde $\lambda_{i}$ son los valores estimados de las raíces características de $\Pi$, y $\mathrm{T}$ es el número de observaciones. Otra alternativa para detectar el número de cointegraciones es el estadístico del máximo eigenvalor, donde la hipótesis nula es de (r) relaciones de cointegración, con una hipótesis alternativa de $r+1$ relaciones de cointegración. Este estadístico está dado por:

$\lambda_{\text {traza }(r, r+1)}=-T \sum_{i=r+1}^{p} L N\left(1-\lambda_{i+1}\right)$

Se realiza la prueba de cointegración de las variables; en la tabla 3 se muestra el resumen de los casos en los que se puede aplicar la prueba de cointegración de Johansen, sin intercepto ni tendencia, con intercepto y sin tendencia, entre otras opciones, además de ofrecer los resultados para los dos criterios disponibles: traza y máximo valor propio. Los resultados arrojan, según el caso y el criterio, entre 1 y 3 relaciones de cointegración, lo cual posibilita inferir que muy probablemente estas variables están cointegradas. Esto hace posible determinar que es factible estimar el modelo VECM, por lo cual se utilizará una relación de cointegración para la estimación del modelo.

Tabla 3.

Prueba de cointegración de Johansen

\begin{tabular}{|c|c|}
\multicolumn{2}{|c|}{$\begin{array}{c}\text { Selected }\left(0.05 \text { level*}^{*}\right) \\
\text { Number of Cointegrating Relations by } \\
\text { Model }\end{array}$} \\
\hline Data Trend: & None intercept no trend \\
\hline Test Type & 3 \\
\hline Trace & 1 \\
\hline Max-Eig & \multicolumn{2}{|c|}{} \\
\hline Critical values based on MacKinnon-Haug-Michelis (1999) \\
\hline \multicolumn{2}{|c|}{} \\
Fuente: elaboración del autor con base en datos del BR de
\end{tabular}

Colombia (2016) y DANE (2017).

\section{Resultados}

El Valle del Cauca se caracteriza por ser un departamento no petrolero, pero sí comercial e industrial; además, es el territorio colombiano que recibe el mayor número de giros de remesas desde el exterior. Por lo anterior, una fluctuación de la tasa de cambio afecta positivamente el consumo de los hogares y la competitividad de la industria del departamento. Las figuras 17 y 18 muestran la respuesta a una desviación estándar del PIB del Valle del Cauca ante un choque en el WTI y el ITCR en los modelos VECM ${ }^{2}$ y $\mathrm{VAR}^{3}$ en niveles. Se observa cómo un impulso de los precios del crudo tiene un efecto negativo y significativo en la actividad económica del departamento. Ello podría darse por la apreciación de la moneda nacional, debido a que un alza en esta induce a la disminución de competitividad de los productos nacionales en el mercado interno frente a la competencia de los productos internacionales; y a ello se suma que los giros de las remesas disminuyen su valor monetario en el país y reducen la capacidad de consumo de los beneficiarios.

Respecto de las importaciones, el modelo permite inferir que un impulso en la ITCR afecta los precios relativos de los productos extranjeros en el mercado nacional, volviéndolos más costosos para los consumidores internos; esto, en consecuencia, genera una reducción en las importaciones nacionales. Las figuras 19 y 20 muestran la respuesta de las importaciones del Valle del Cauca frente a innovaciones en el precio del petróleo WTI y el ITCR a una desviación estándar. Como se puede observar, un choque positivo en el WTI genera una respuesta positiva a las importaciones del Valle del Cauca, pero solo es significativo en el quinto y décimo mes para el modelo VAR.

2 El programa Eviews no arroja intervalos de confianza para el modelo VEC.

3 Se utiliza un modelo VECM (12) y VAR (8), donde se corrige autocorrelación, normalidad y heteroscedasticidad. Se establece la variable proxy de la tasa de interés como totalmente exógena, y una variable dummy para atenuar la crisis económica del 2008. 
Gráfica 17.

Respuesta PIB del Valle del Cauca ante un choque en ITCR y WTI, VECM

Response to Cholesky One S.D. Innovations
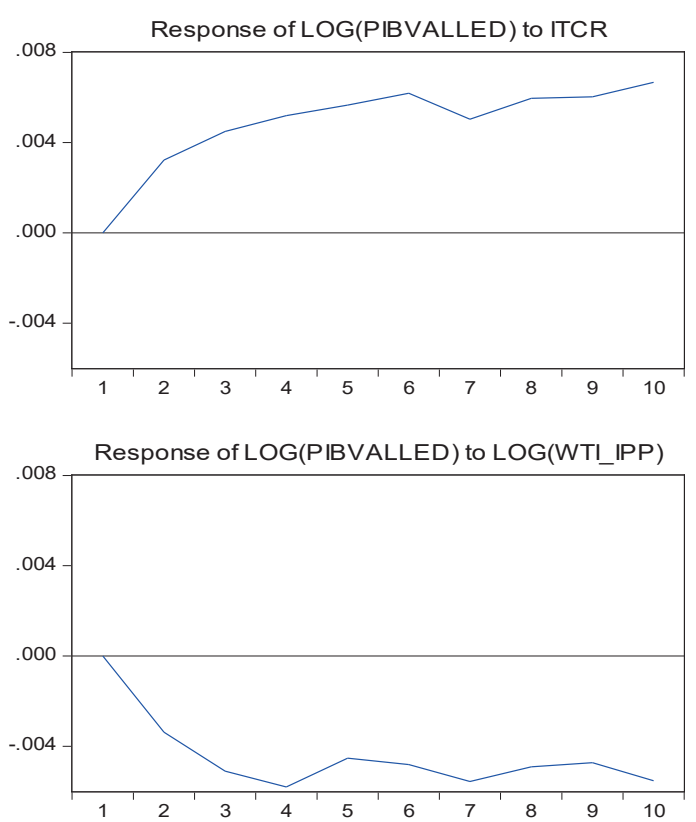

Gráfica 18.

Respuesta PIB del Valle del Cauca ante un choque en ITCR y WTI, VAR niveles

Response to Cholesky One S.D. Innovations \pm 2 S.E. Response of LOG(PIBVALLED) to ITCR

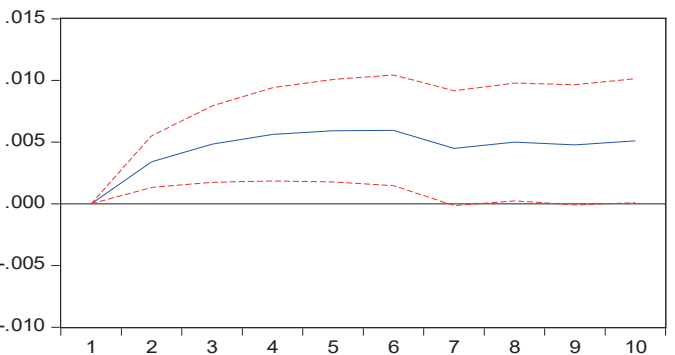

Response of LOG(PIBVALLED) to LOG(WTI_IPP)

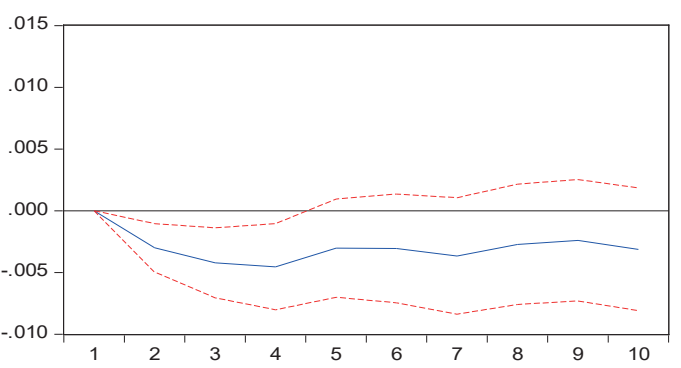

Fuente: elaboración del autor con base a información Banco de la Republica y el DANE.
Por otro lado, la respuesta de las importaciones del Valle del Cauca frente a un impuso en el ITCR produce un efecto opuesto y significativo desde el tercer mes, en comparación con lo mostrado por el WTI. Los resultados de las figuras 19 y 20 se deben a que el incremento de los precios del petróleo genera un aumento considerable en el ingreso de divisas al interior del país; en consecuencia, se aprecia la moneda nacional, en tanto los precios relativos de los productos extranjeros disminuyen, causando una mayor entrada de dichos productos al país y al departamento.

Gráfica 19.

Respuesta de las importaciones ante una innovación en ITCR y WTI, VECM

Response to Cholesky One S.D. Innovations Response of LOG(IMPO) to ITCR

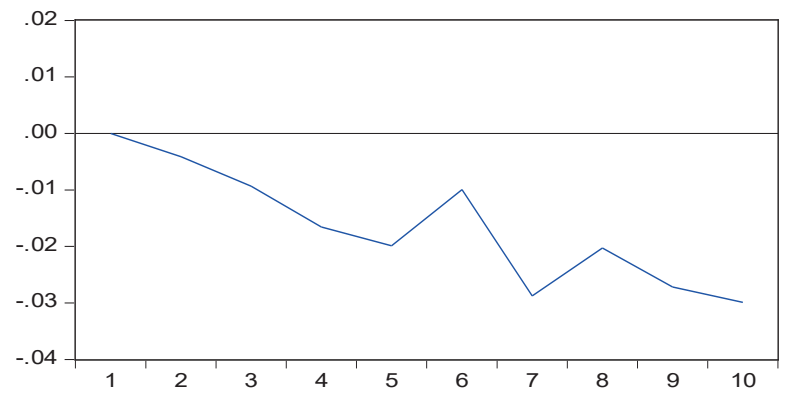

Response of LOG(IMPO) to LOG(WTI_IPP)

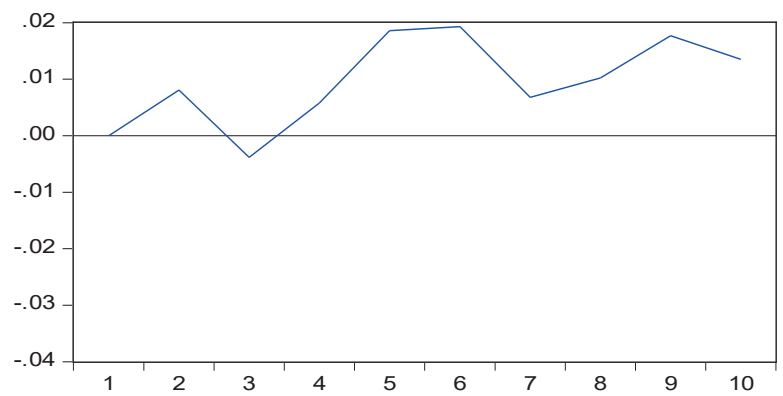

De igual forma, se analizó el comportamiento de las exportaciones cuando hay un impulso de los precios del petróleo y la tasa de cambio real. Las gráficas 21 y 22 presentan la respuesta de las exportaciones ante un impulso del ITCR y el WTI a una desviación estándar; se observa allí que un aumento en los precios del petróleo genera un impacto positivo y significativo en las exportaciones en los 
Gráfica 20.

Respuesta de las importaciones ante una innovación en ITCR y WTI, VAR niveles

Response to Cholesky One S.D. Innovations \pm 2 S.E. Response of LOG(IMPO) to ITCR

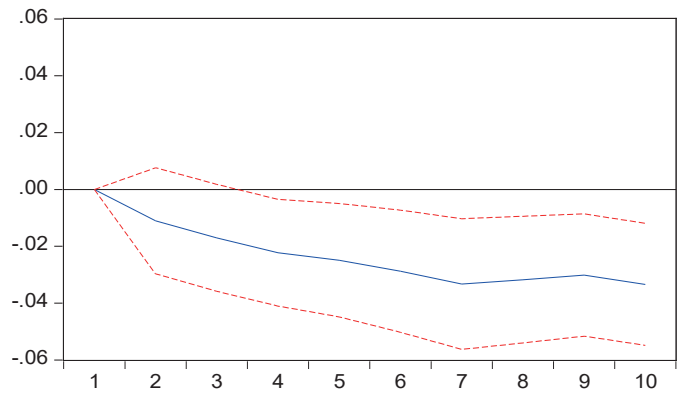

Response of LOG(IMPO) to LOG(WTI_IPP)

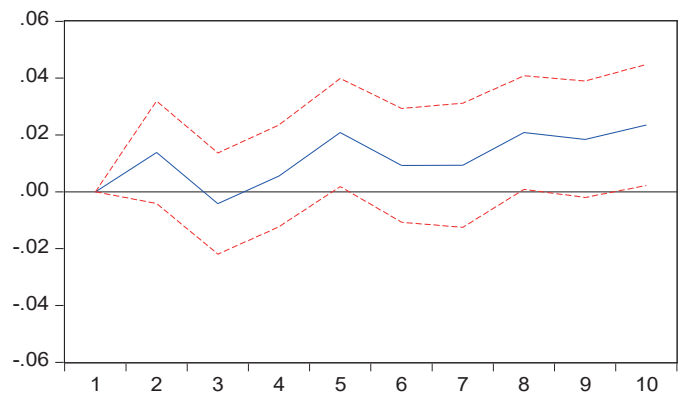

Fuente: elaboración del autor con base a información Banco de la Republica de Colombia y el DANE.

periodos 1, 2, 8 y 10 para el modelo VAR. Para el modelo VECM se tienen los mismos efectos para los mismos periodos, aunque no es claro que aquellos sean positivos en todos los meses. Esto se debe a que un alza en la cotización del WTI provoca una revaluación de la moneda interna, y puesto que la industria del Valle del Cauca usa materias primas y tecnología importada, tiene lugar una reducción de costos por el abaratamiento relativo de los productos extranjeros.

Asimismo, en las figuras 21 y 22 se puede observar que un impulso en la tasa de cambio real genera impactos negativos para los dos modelos, y en relación con el modelo VAR, los choques son significativos para el cuarto y séptimo mes. Esto podría deberse a que se transmite un aumento de los costos de insumos importados para la industria del Valle del Cauca. Dado el efecto del ITCR y el WTI en las exportaciones vallecaucanas, podría inferirse que el efecto de reducción de costos debido a la revaluación de la moneda que genera un abaratamiento de los insumos debe ser mayor que el efecto que tiene la revaluación de la moneda interna frente a la competitividad internacional ${ }^{4}$.

Gráfica 21.

Respuesta de las exportaciones frente a un choque en ITCR y WTI, VEMC

Response to Cholesky One S.D. Innovations Response of LOG(EXPORTACIONES) to ITCR

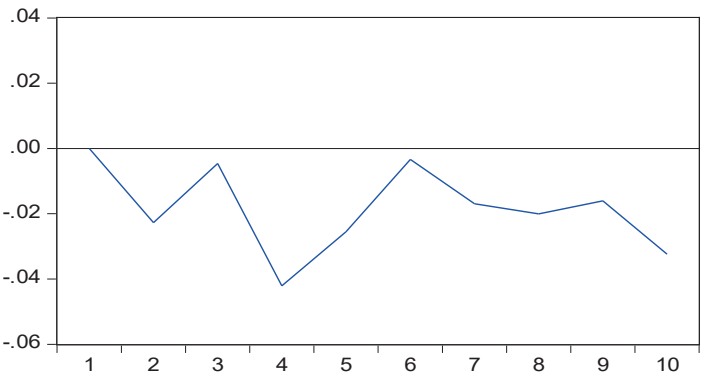

Response of LOG(EXPORTACIONES) to LOG(WTI_IPP)

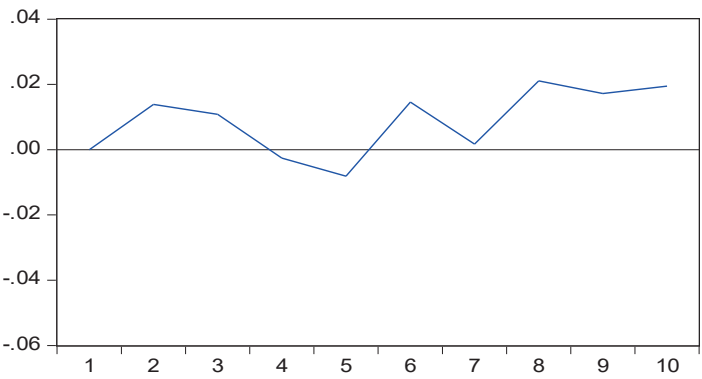

Fuente: elaboración del autor

Por otro lado, con el fin de confirmar cuál es el efecto de un alza de los precios del petróleo con respecto a la economía colombiana, las figuras 23 y 24 muestran la respuesta del indicador de seguimiento económico para Colombia (ISE) frente a un impacto positivo en el precio del petróleo (WTI) a una desviación estándar. Ello confirma lo encontrado por Uribe y Ulloa (2011) y González y Hernández (2016), que identifican una relación positiva entre el

$4 \quad$ Se debe resaltar que solo se desincentiva la exportación de productos, pero no su compra en el mercado interno, ya que, aunque exista un encarecimiento de los productos que necesitan maquinaria y materias primas importadas, igualmente sucede con la importación de productos para el consumo. La compra de productos del Valle del Cauca se ve dinamizado por la correlación de la economía departamental con la nacional. 
Gráfica 22.

Respuesta de las exportaciones frente a un choque en ITCR y WTI, VAR niveles

Response to Cholesky One S.D. Innovations \pm 2 S.E. Response of LOG(EXPORTACIONES) to ITCR

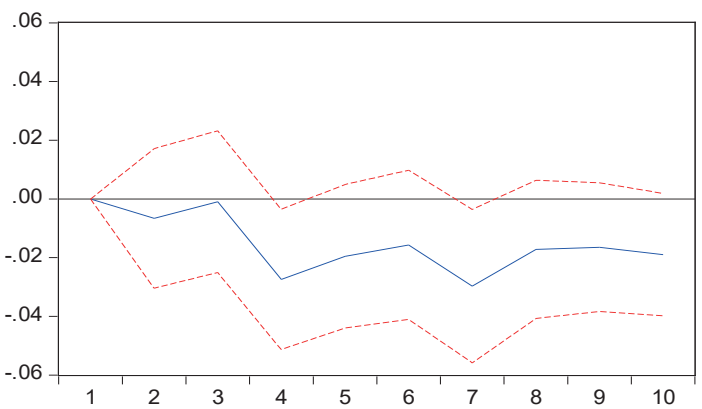

Response of LOG(EXPORTACIONES) to LOG(WTI_IPP)

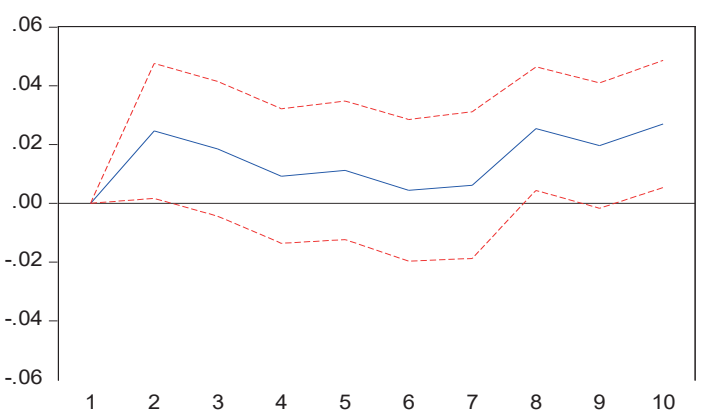

Fuente: elaboración del autor con base a información Banco de la Republica de Colombia y el DANE.

aumento de los precios del petróleo y el crecimiento del PIB de Colombia, donde para el modelo VAR los impactos son significativos en los periodos 4 y 5 .

Gráfica 23.

Respuesta del ISE frente a un choque del WTI, VECM

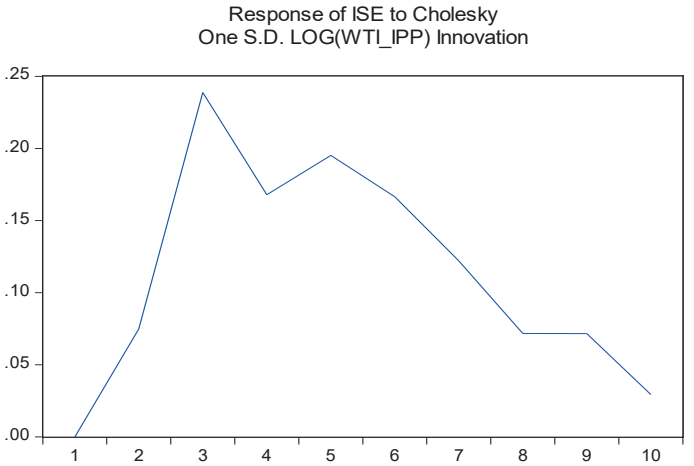

Fuente: elaboración del autor con base a información Banco de la Republica de Colombia y el DANE.
Gráfica 24.

Respuesta del ISE frente a un choque del WTI, VAR niveles

Response of ISE to Cholesky One S.D. LOG(WTI_IPP) Innovation

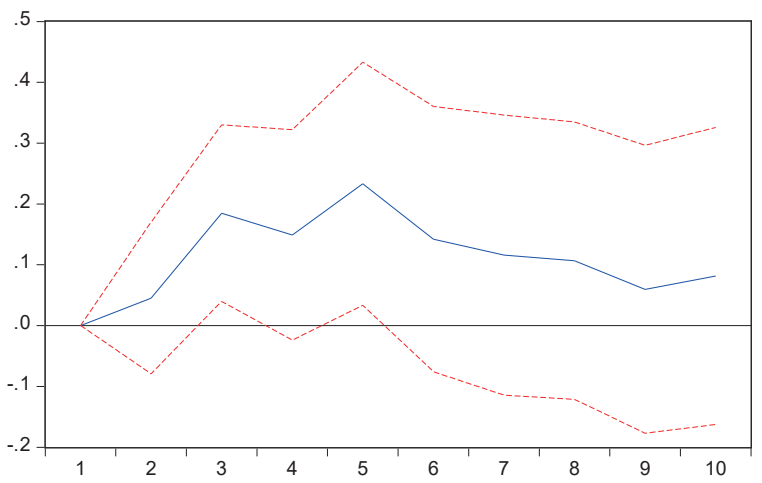

Fuente: elaboración del autor con base a información Banco de la Republica de Colombia y el DANE.

\section{Efectos asimétricos}

La literatura ha identificado que los impactos del precio del petróleo pueden ser asimétricos. Así, Kilian y Vigfusson (2009) encuentran para Estados Unidos una asimetría en la respuesta frente a los cambios de los precios del crudo; sostiene que los choques positivos generan un impacto mucho mayor que los negativos, lo cual reafirma estudios como los de Hamilton (2000), Lee, Ni y Ratti (1995), Davis y Haltiwanger (2001), Bernanke (1983), Hamilton (1988), Pindyck (1991) y Balke, Brown y Yücel (2002). Para algunas economías latinoamericanas, ciertos autores también identifican asimetría en los choques del hidrocarburo; entre ellos están Mendoza y Vera (2010) para Venezuela, y Perrilla (2009), Uribe y Ulloa (2011) y González y Hernández (2016) para Colombia.

En este trabajo también se identificaron los choques asimétricos de los precios del crudo en la economía vallecaucana. Para resolver este problema, autores como Mork (1989) y Hamilton (2003) usan una trasformación de la variable para identificar los efectos de los grandes cambios positivos en el precio del petróleo, lo cual es especificado de la siguiente forma:

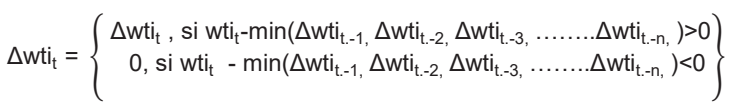


Por su parte, Mork, Øystein y Mysen (1994) establecen que la asimetría y el efecto de los precios positivos se puede identificar con la siguiente transformación de la serie de los precios del petróleo:

$\Delta w t_{t}=\left\{\begin{array}{c}\Delta w i_{t}, \text { si } \Delta w t_{i}>0 \\ 0, \text { si } \Delta w t_{t}<0\end{array}\right\}$

Por último, para identificar los efectos de los choques negativos, según Kiliam (2009), se puede usar la siguiente transformación:

$\Delta w t_{t}=\left\{\begin{array}{c}\Delta w t i, \text { si } \Delta w t i<0 \\ 0, \text { si } \Delta w t i>0\end{array}\right\}$

Para el Valle del Cauca se identifica que sí existen efectos asimétricos entre los impactos negativo o positivo de los precios del petróleo; para ello se usó la metodología VAR en niveles, lo cual permitió identificar que el aumento de los precios del petróleo tiene un impacto mayor que una baja de este. Como se puede identificar en las figuras 25 y 26, la demanda agregada del Valle del Cauca, al aumentar el precio del petróleo, tiene un efecto negativo por cinco periodos. Por otro lado, una baja de los precios del hidrocarburo genera un aumento a la demanda agregada en todos los periodos, pero solo en el periodo 5 este es significativo. Para las exportaciones e importaciones no se identificaron efectos significativos.

\section{Descomposición de varianza}

Las tablas 4 y 5 muestran la descomposición de varianza para ambos modelos, lo cual permite identificar qué tanto afecta el cambio de una variable sobre la fluctuación de otra. En principio, se puede observar que la mayoría de la variabilidad de la demanda agregada del Valle del Cauca está dada por los rezagos de ella misma, debido a que el presente de la demanda agregada del Valle está explicada hasta por un $100 \%$ por el primer periodo de su pasado. Un dato interesante en los dos modelos es que la tasa de cambio puede explicar en gran medida las fluctuaciones de la actividad económica vallecaucana ( $18 \%$ modelo VECM), y de igual forma lo hacen las fluctuaciones del precio del petróleo.

Funciones de impulso-respuesta de efectos asimétricos ante un choque positivo del WTI, VAR niveles
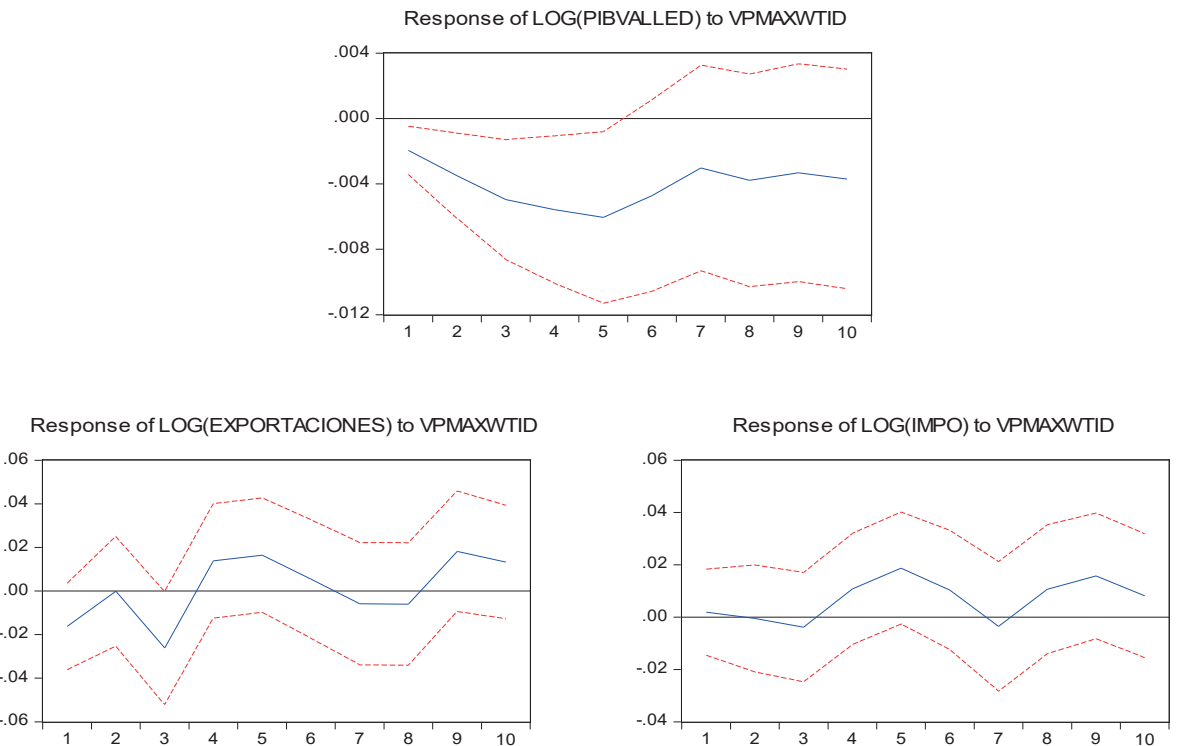

Fuente: elaboración del autor con base a información Banco de la Republica de Colombia y DANE. 
Funciones de impulso-respuesta de efectos asimétricos ante un choque negativo del WTI, VAR niveles Response to Cholesky One S.D. Innovations \pm 2 S.E. Response of LOG(PIBVALLED) to VNWTID
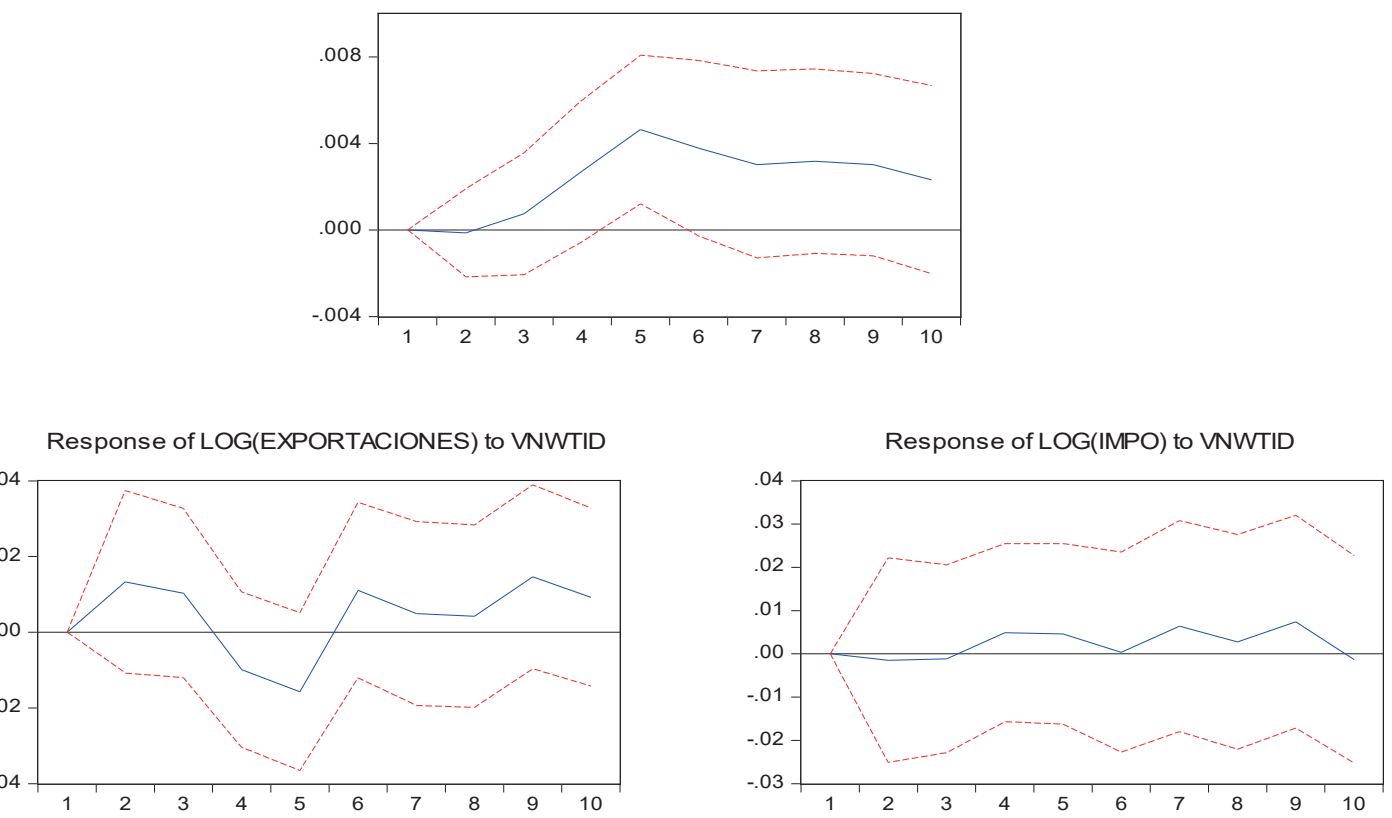

Fuente: elaboración del autor con base a información Banco de la Republica de Colombia y DANE.

Tabla 4.

Descomposición de varianza VECM

\begin{tabular}{|c|c|c|c|c|c|c|c|}
\hline \multicolumn{7}{|c|}{ Variance Decomposition of LOG(PIBVALLD): } \\
\hline Period & S.E & LOG(PIBVA $\ldots$ & ISE & LOG(EXPO $\ldots$ & LOG(IMPO) & ITCR & LOG(WTI_IP... \\
\hline 1 & 0,009816 & 100,0000 & 0,000000 & 0,000000 & 0,000000 & 0,000000 & 0,000000 \\
\hline 2 & 0,015415 & 90,00931 & 0,444211 & 0,352390 & 0,005066 & 4,382314 & 4,806709 \\
\hline 3 & 0,020045 & 82,05486 & 0,746775 & 0,239380 & 0,019429 & 7,614150 & 9,325409 \\
\hline 4 & 0,023944 & 76,17415 & 0,920877 & 0,228093 & 0,225811 & 10,04628 & 12,40479 \\
\hline 5 & 0,027597 & 72,10797 & 2,646619 & 0,243628 & 1,196544 & 11,77397 & 12,03127 \\
\hline 6 & 0,030761 & 69,43384 & 2,942462 & 0,210749 & 1,766158 & 13,51849 & 12,12831 \\
\hline 7 & 0,033210 & 66,79575 & 3,233476 & 0,230656 & 2,636403 & 13,89528 & 13,20843 \\
\hline 8 & 0,034947 & 64,11782 & 3,431651 & 0,560762 & 2,530186 & 15,45801 & 13,90157 \\
\hline 9 & 0,036281 & 60,55557 & 4,063317 & 1,278626 & 2,406532 & 17,09948 & 14,59648 \\
\hline 10 & 0,037837 & 55,86794 & 5,147429 & 2,254537 & 2,346425 & 18,82859 & 15,55509 \\
\hline
\end{tabular}

Fuente: elaboración del autor con base a información Banco de la Republica de Colombia y el DANE. 
Tabla 5.

Descomposición de varianza VAR niveles

\begin{tabular}{|c|c|c|c|c|c|c|c|}
\hline \multicolumn{9}{|c|}{ Variance Decomposition of LOG(PIBVALLED): } \\
\hline Periodo & S.E & LOG(PIBVA $\ldots$ & ISE & LOG(EXPO $\ldots$ & LOG(IMPO) & ITCR & LOG(WTI_IP... \\
\hline 1 & 0,009304 & 100,0000 & 0,000000 & 0,000000 & 0,000000 & 0,000000 & 0,000000 \\
\hline 2 & 0,014506 & 88,57068 & 0,865343 & 0,282393 & 0,108877 & 5,459202 & 4,713502 \\
\hline 3 & 0,019322 & 75,39987 & 1,518671 & 0,215897 & 0,725192 & 12,56889 & 9,571481 \\
\hline 4 & 0,023252 & 66,36490 & 1,551084 & 0,341471 & 1,236126 & 17,91483 & 12,59159 \\
\hline 5 & 0,026322 & 60,66481 & 2,502316 & 0,363695 & 1,210506 & 22,34482 & 12,91386 \\
\hline 6 & 0,028970 & 56,16260 & 2,528420 & 0,301094 & 1,509584 & 26,03140 & 13,46690 \\
\hline 7 & 0,031118 & 52,42982 & 2,634331 & 0,322514 & 1,468909 & 28,48725 & 14,65717 \\
\hline 8 & 0,032761 & 48,76922 & 2,674759 & 0,296542 & 1,786220 & 31,03972 & 15,43354 \\
\hline 9 & 0,034057 & 45,66614 & 2,616843 & 0,316020 & 1,875998 & 33,29707 & 16,22794 \\
\hline 10 & 0,035021 & 43,38183 & 2,560285 & 0,545511 & 1,885251 & 35,02732 & 16,59980 \\
\hline
\end{tabular}

Fuente: elaboración del autor con base a información Banco de la Republica de Colombia y el DANE.

\section{REFLEXIONES FINALES}

El presente estudio tuvo como objetivo identificar cuáles son los efectos de los precios del petróleo y la tasa de cambio en la economía del Valle del Cauca, Colombia, además de indagar cuánto persiste un choque de dichas variables en la economía de este departamento. Para ello, se utilizaron un vector de corrección de errores (VECM) y un vector autorregresivo (VAR) en niveles para el periodo comprendido entre enero de 2001 y diciembre 2015.

Los resultados del proceso de estimación permiten inferir que un impulso de los precios del barril del petróleo West Texas Intermediate (WTI) —que es referencia para Colombia-impactan negativa y significativamente la demanda agregada del departamento del Valle del Cauca. Igualmente, existe un efecto asimétrico frente a una subida o una baja del precio del crudo, donde un aumento del precio del crudo tiene mayores efectos que una disminución de este. Lo anterior puede ser resultado de que el departamento presenta una actividad económica que no gira en torno al petróleo, sino que está en función del sector industrial y comercial. Sin embargo, no se podría afirmar que el Valle del Cauca padece de enfermedad holandesa, a pesar del alto ingreso de divisas ocasionada por la exportación de bienes minero-energéticos de Colombia.

La economía vallecaucana se identifica por ser el territorio colombiano que recibe el mayor número de remesas desde el exterior; por ello, es posible inferir que el departamento sufre un doble impacto al producirse una revaluación de la moneda interna por el aumento de los precios de petróleo (WTI). El primero es una pérdida de competitividad por parte de la industria en el mercado interno y el segundo es la disminución del poder adquisitivo por parte de las familias receptoras de remesas desde el exterior, que se estiman en poco más de 1500 millones de dólares (DANE, 2017).

Por otro lado, las estimaciones realizadas muestran que un impulso en el índice de tasa de cambio real, tomando como base el dólar estadounidense frente al peso colombiano, genera un choque positivo sobre la economía del Valle del Cauca, lo que podría explicarse por la pérdida de competitividad de los productos internacionales, sumado a un 
aumento del poder adquisitivo de las familias que reciben remesas del extranjero.

Aunque las importaciones y exportaciones se ven afectadas negativamente por una devaluación de la moneda nacional, hay que tener en cuenta que el impacto en las importaciones con relación al PIB del Valle del Cauca es mucho mayor que el de las exportaciones, ya que existe un gran déficit comercial; y aunque una revaluación del dólar encarezca las materias primas de los productos nacionales, sucede los mismo con los precios de los productos internacionales, y así es posible que la industria departamental se concentre en el desarrollo del mercados nacionales.
Por último, se resalta que los resultados encontrados en esta investigación evidencian un impacto contrario de la variable precios del petróleo en el departamento y el país, puesto que autores como Uribe y Ulloa (2011), González y Hernández (2016) y Gómez (2015) identificaron efectos positivos en la economía nacional cuando aquellos aumentaban. Lo anterior permite identificar que existen efectos diferenciados en el auge del sector minero-energético para las distintas regiones del país, y por ello surge el interrogante de si las políticas deben de ser diferenciadas para cada una de ellas.

\section{REFERENCIAS}

1. Alonso, J. y Patiño, C. (2007). ¿Crecer para exportar o exportar para crecer? El caso del Valle del Cauca. Ensayos sobre Economía Regional, 46. Recuperado de http://www.banrep.gov.co/sites/default/files/publicaciones/archivos/2007_julio_a.pdf

2. Arbeláez, A. Espacio, A. y Olivera, M. (2009). Impacto socioeconómico del sector azucarero colombiano en la economía nacional y regional. Bogotá: Fedesarrollo.

3. Banco de la República (2017). Distribución de los ingresos de remesas por país de origen y departamento de destino. Bogotá: Autor.

4. Balke, N. S., Brown, S. y Yücel, M. (2002). Oil price shocks and the U.S. Economy: Where does the asymmetry originate? Energy Journal, 23(3), 27-52. Recuperado de https:/www.jstor.org/ stable/41322962?seq=1\#page_scan_tab_contents

5. Bernanke, B. S. (1983). Irreversibility, uncertainty, and cyclical investment. Journal of Economics, 98, 85-106.

6. Biørnland, H. C. (2009). Oil price shocks and stock market booms in an oil exporting country. Scottish Journal of Political Economy, 56(2), 232-53.

7. Cámara de Comercio de Cali (2016). Informe Económico \# 72: Enfoque competitivo. Santiago de Cali: Autor.

8. Cámara de Comercio de Cali (2017). Informe Económico \# 23: Ritmo exportador. Santiago de Cali: Autor.

9. Corden, W. M. (1984). Booming sector and Dutch disease economics: Survey and consolidation. Oxford Economic Papers, 36(3), 359-380.

10. Departamento Administrativo Nacional de Estadística (DANE) (2016). Boletín técnico, Cuentas Departamentales - Colombia Producto Interno Bruto (PIB) 2016. Recuperado de https://www.dane.gov. co/files/investigaciones/pib/departamentales/B_2005/Bol dptal 2016preliminar.pdf

11. Departamento Administrativo Nacional de Estadística (DANE) (2016). Boletín técnico Encuesta Anual Manufacturera (EAM). Bogotá: Autor.

12. Davis, S. J. y Haltiwanger, J. (2001). Sectoral job creation and destruction responses to oil price changes. Journal of Monetary Economics, 48(3), 465-512. 
13. Dickey, D. A. y Fuller, W. A. (1979). Distribution of the estimators for autoregressive time series with a unit root. Journal of the American Statistical Association, 74(366), 427-431.

14. Douglas, C. N. (1990). Cambio institucional y desempeño económico. Ciudad de México: Fondo de Cultura Económica.

15. Echavarría, J. J., Faimboin, I. y Zuleta, L. A. (2002). Explicaciones de la crisis de la economía vallecaucana y posibles elementos para su recuperación. Bogotá: Fedesarrollo.

16. Égert, B. y Leonard, C. (2008). The Dutch disease in Kazakhstan: An empirical investigation. Recuperado de https://ideas.repec.org/a/onb/oenbfi/y2006i2b3.html

17. Engle, R.F. y Granger, C. W. J. (1987). Co-integration and error correction: Representation, estimation, and testing. Econometrica, 55(2), 251-276.

18. Frenkel, R. y Ros, J. (2006). Unemployment and the real exchange rate in Latin America. World Development, 34(4), 631-646.

19. Gómez, C. (2015). Más allá de un boom de recursos naturales: efectos de los choques petroleros en la economía colombiana. Recuperado de https://ideas.repec.org/p/col/000089/012565.html

20. Gómez, J. E. y Morales, P. (2009). Bank lending channel of monetary policy: Evidence for Colombia, using a firms' panel Data. Borradores de Economía, 545, 1-13. Recuperado de: http://www.banrep.gov.co/es/node/196

21. Gómez, J., Uribe, J. D. y Vargas, H. (2002). The implementation of inflation targeting in Colombia. Borradores de Economía, 202.

22. González, S. y Hernández, E. (2016). Impactos indirectos de los precios del petróleo en el crecimiento económico colombiano. Lecturas de Economía, 84, 103-141.

23. Hamilton, J. D. (1983). Oil and the Macroeconomy since World War II. The Journal of Political Economy, $91(2), 228-248$.

24. Hamilton, J. D. (2003). What is an oil shock? Journal of Econometrics, 113(2), 363-398.

25. Hernandez, E. y Raffo, L. (2016). Comercio intra-industrial y costos de ajuste para la industria del Valle del Cauca (1975-2014). Semestre Económico, 19(41), 137-166.

26. Hernández, G. (2013). Colombia: términos de intercambio y fluctuaciones de la producción. Revista CEPAL, 110, 109-131.

27. Hodrick, R. y Prescott, E. C. (1980). Postwar U.S. business cycles: An empirical investigation [Discussion Papers 451]. Pittsburgh: Universidad Carnegie-Mellon.

28. Hua, P. (2007). Real exchange rate and manufacturing employment in China. China Economic Review, $18(3), 335-353$

29. Jin, J. C. (2002). Exports and growth: Is the export-led growth hypothesis valid for provincial economies? Applied Economics Letters, 34, 63-76.

30. Johansen, S. (1988). Statistical analysis of cointegration vectors. Journal of Economic Dynamics and Control, $12,231-254$.

31. Johansen, S. (1991). Estimation and hypothesis testing of cointegration vectors in gaussian vector autoregressive models. Econometrica, 59, 1551-1580.

32. Johansen, S. (1995). Likelihood-based inference in cointegrated vector autoregressive models. Nueva York: Oxford University Press. 
33. Kilian, L. y Vigfusson, R. (2011). Are the responses of the U.S. Economy asymmetric in energy price increases and decreases? Míchigan: Universidad de Míchigan. Recuperado de http://wwwpersonal.umich.edu/ lkilian/ kvsubmission.pdf

34. Kose, M. A. y Riezman, R. G. (1999). Trade shocks and macroeconomic fluctuations in Africa [Working Paper 203]. Múnich: Center for Economic Studies e Institute for Economic Research.

35. Lartey, E (2008). Capital inflows, resource reallocation and the real exchange rate. International Finance, $11(2), 131-152$.

36. Lee, K., Ni, S. y Rati, R. A. (1995). Oil shocks and macroeconomy: The role of price variability. The Energy Journal, 16(4), 39-56.

37. Leung, D. y T. Yuen (2007). Labour market adjustments to exchange rate fluctuations: Evidence from Canadian manufacturing industries. Open Economies Review, 18, 177-189.

38. Martínez, H. (2006). Agroindustria y competitividad, estructura y dinámica Colombia 1992-2006. Recuperado de bibliotecadigital.agronet.gov.co/bitstream/11348/6372/1/179.pdf

39. Mendoza, O. y Vera, D. (2010). The asymmetric effects of oil shocks on an oil-exporting Economy. Cuadernos de Economía, 47(135), 3-13.

40. Mork, K. A. (1989). Oil and the macroeconomy when prices go up and down: An extension of Hamilton's results. Journal of Political Economy, 97(3), 740-744.

41. Mork, K. A., Olsen, Ø. y Mysen, H. T. (1994). Macroeconomic responses to oil price increases and decreases in seven OECD countries. The Energy Journal, 15(4), 19-35.

42. Peláez, J. T. y Sierra, L. P. (2016). Does industrial employment react to movements in the real exchange rate? An empirical analysis for Colombia, 2000-2010. Latin American Journal of Economics, 53(1), 39-60. https://dx.doi.org/10.7764/LAJE.53.1.39

43. Perilla, J. R. (2010). Descomposición sectorial y dinámica del impacto de los precios del petróleo sobre el crecimiento económico en Colombia. Bogotá: Departamento Nacional de Planeación.

44. Pesaran, M. H., Shin, Y. y Smith, R. (2000). Structural analysis of vector error correction models with exogenous i(1) variables. Journal of Econometrics, 97, 293-343.

45. Phillips, P. C. y Ouliaris, S. (1990). Asymptotic properties of residual based tests for cointegration. Econometrica, 58(1), 165-193.

46. Phillips, C. B. (1988). Regression theory for near-integrated time series. Econometrica, 56(5), 1021-1043.

47. Pindyck, R.S. (1991). Irreversibility, Uncertainty, and investment. Journal of Economic Literature, 29, $1110-1148$

48. Poncela, P., Senra, E. y Sierra, L. P. (2016). Long-term links between raw materials prices, real exchange rate and relative de-industrialization in a commodity-dependent economy: empirical evidence of "Dutch disease" in Colombia. Empirical Economics, 52(2), 777-798.

49. Red de Observatorios Regionales del Mercado de Trabajo (ORMET) (2012). Estructura socioeconómica del Valle del Cauca: un análisis del mercado laboral de Cali y su Área Metropolitana. Recuperado de http://www.observatoriovalle.org.co/wp-content/uploads/2012/05/Estructura-Valle-del-Cauca.pdf

50. Ricci, A. y Ramojo, J (2012). Un modelo VECMX* con variables fiscales para la economía española. Extremadura: Universidad de Extremadura. 
51. Sierra, L. P. y Manrique, K. (2014). Impacto del tipo de cambio real en los sectores industriales de Colombia: una primera aproximación. Recuperado de https://repositorio.cepal.org/bitstream/handle/11362/37440/1/ RVE114SierraManrique_es.pdf

52. Sims, C. A. (1980). Macroeconomics and reality. Econometrica, 48, 1-48.

53. Sims, C. A., Stock, J. H. y Watson, M. A. (1990). Inference in linear time series models with some unit roots. Econometrica, 58(1), 113-144.

54. Tokarick, S. (1995). External shocks, the real exchange rate and tax policy. IMF Staff Papers, 42, 49-79.

55. Uribe, J. y Ulloa, I. (2011). Otro país exportador neto de petróleo y sus reacciones macroeconómicas ante cambios del precio: Colombia [Documentos de Trabajo, 140]. Cali: Universidad del Valle.

56. Vargas, H., Hamann, F. y González, A. (2010). Efectos de la política monetaria sobre las tasas de interés de los créditos hipotecarios en Colombia. Borradores de Economía, 592, 1-11. Recuperado de http://www. banrep.gov.co/es/borrador-592 


\section{ANEXOS}

\section{Anexo 1. Estadísticos de prueba LM multivariadas para la correlación serial residual hasta el orden especificado}

Hipótesis nula: no hay correlación serial del orden $\mathrm{h}$, la estadística LM está distribuida asintóticamente $\mathrm{x}_{2}$ con $\mathrm{k}_{2}$ grados de libertad.

VEC Residual Serial Correlation LM Te...

Null Hypothesis: no serial correlation at...

Date: 05/24/18 Time: 11:29

Sample: 2001M01 2015M12

Included observations: 167

\begin{tabular}{|c|c|c|}
\hline Lags & LM-Stat & Prob \\
\hline 1 & 42,01720 & 0,2264 \\
\hline 2 & 43,21638 & 0,1903 \\
\hline 3 & 36,75267 & 0,4338 \\
\hline 4 & 36,34342 & 0,4527 \\
\hline 5 & 28,62668 & 0,8041 \\
\hline 6 & 28,30986 & 0,8160 \\
\hline 7 & 38,14236 & 0,3722 \\
\hline 8 & 37,69009 & 0,3918 \\
\hline 9 & 46,54185 & 0,1121 \\
\hline 10 & 32,72277 & 0,6253 \\
\hline 11 & 39,83653 & 0,3033 \\
\hline 12 & 42,41465 & 0,2139 \\
\hline 13 & 36,00474 & 0,4684 \\
\hline
\end{tabular}

Probs from chi-square with $36 \mathrm{df}$.

Fuente: elaboración del autor.

\section{Anexo 2. Prueba de heterocedasticidad de White}

Hipótesis nula: sin heterocedasticidad o (sin especificación incorrecta).

VEC Residual Heteroskedasticity Tests: No Cross Terms (only levels and squares)

Date: 05/24/18 Time: 11:29

Sample: 2001M01 2015M12

Included observations: 167

\begin{tabular}{|c|c|c|}
\hline Join test: & \multicolumn{2}{|c|}{} \\
\hline Chi-sq & df & Prob. \\
\hline 3184,065 & 3129 & 0,2418 \\
\hline
\end{tabular}

Fuente: elaboración del autor. 


\section{Anexo 3. Prueba de normalidad de residuales Jarque-Bera modelo VEC}

Hipótesis nula: los residuos son normales multivariados.

VEC Residual Normality Tests

Orthogonalization: Cholesky (Lutkepohl)

Null Hypothesis: residuals are multivariate normal

Date: 05/24/18 Time: 11:31

Sample: 2001M01 2015M12

Included observations: 167

\begin{tabular}{|c|c|c|c|c|}
\hline Component & Skewness & Chi-sq & df & Prob. \\
\hline 1 & $-0,021240$ & 0,012557 & 1 & 0,9108 \\
\hline 2 & $-0,097392$ & 0,264005 & 1 & 0,6074 \\
\hline 3 & $-0,104673$ & 0,304955 & 1 & 0,5808 \\
\hline 4 & 0,126633 & 0,446335 & 1 & 0,5041 \\
\hline 5 & 0,150223 & 0,628115 & 1 & 0,4280 \\
\hline 6 & 0,129011 & 0,463251 & 1 & 0,4961 \\
\hline Joint & & 2,119217 & 6 & 0,9084 \\
\hline Component & Kurtosis & Chi-sq & df & Prob. \\
\hline 1 & 3,630039 & 2,762108 & 1 & 0,0965 \\
\hline 2 & 3,030051 & 0,006284 & 1 & 0,9368 \\
\hline 3 & 2,740066 & 0,470144 & 1 & 0,4929 \\
\hline 4 & 3,426231 & 1,264142 & 1 & 0,2609 \\
\hline 5 & 3,207718 & 0,300229 & 1 & 0,5837 \\
\hline 6 & 2,865514 & 0,125851 & 1 & 0,7228 \\
\hline Joint & & 4,928758 & 6 & 0,5530 \\
\hline
\end{tabular}

\begin{tabular}{|c|c|c|c|}
\hline Component & Jarque-Bera & $\mathrm{df}$ & Prob. \\
\hline 1 & 2,774665 & 2 & 0,2497 \\
\hline 2 & 0,270288 & 2 & 0,8736 \\
\hline 3 & 0,775099 & 2 & 0,6787 \\
\hline 4 & 1,710476 & 2 & 0,4252 \\
\hline 5 & 0,928343 & 2 & 0,6287 \\
\hline 6 & 0,589103 & 2 & 0,7449 \\
\hline Joint & 7,047975 & 12 & 0,8544 \\
\hline
\end{tabular}

Fuente: elaboración del autor. 


\section{Anexo 4. Raíces del polinomio característico VECM}

Roots of Characteristic Polynomial

Endogenous variables: LOG(PIBVALLED) ISE LOG(E...

Exogenous variables: TASA_DE_INTERES_MUNDIA...

Lag specification: 112

Date: 05/24/18 Time: 11:31

\begin{tabular}{|c|c|c|}
\hline 1,000000 & & 1,000000 \\
\hline 1,000000 & $-1,87 e-15 i$ & 1,000000 \\
\hline 1,000000 & $+1,87 \mathrm{e}-15 \mathrm{i}$ & 1,000000 \\
\hline 1,000000 & & 1,000000 \\
\hline 1,000000 & & 1,000000 \\
\hline 0,986889 & & 0,986889 \\
\hline$-0,021874$ & $+0,969471 \mathrm{i}$ & 0,969718 \\
\hline$-0,021874$ & $-0,969471 i$ & 0,969718 \\
\hline 0,373559 & $+0,884758 \mathrm{i}$ & 0,960387 \\
\hline 0,373559 & $-0,884758 \mathrm{i}$ & 0,960387 \\
\hline$-0,851850$ & $+0,437280 \mathrm{i}$ & 0,957530 \\
\hline$-0,851850$ & $-0,437280 \mathrm{i}$ & 0,957530 \\
\hline 0,445467 & $+0,845994 \mathrm{i}$ & 0,956110 \\
\hline 0,445467 & $-0,845994 i$ & 0,956110 \\
\hline 0,843038 & $-0,449937 i$ & 0,955592 \\
\hline 0,843038 & $+0,449937 i$ & 0,955592 \\
\hline$-0,535128$ & $-0,773516 i$ & 0,940579 \\
\hline$-0,535128$ & $+0,773516 i$ & 0,940579 \\
\hline 0,592538 & $+0,727142 i$ & 0,937996 \\
\hline 0,592538 & $-0,727142 \mathrm{i}$ & 0,937996 \\
\hline$-0,486515$ & $+0,801135 i$ & 0,937291 \\
\hline$-0,486515$ & $-0,801135 i$ & 0,937291 \\
\hline 0,893138 & $+0,279569 \mathrm{i}$ & 0,935871 \\
\hline 0,893138 & $-0,279569 i$ & 0,935871 \\
\hline 0,707960 & $+0,606540 \mathrm{i}$ & 0,932254 \\
\hline 0,707960 & $-0,606540 \mathrm{i}$ & 0,932254 \\
\hline$-0,808388$ & $+0,460529 i$ & 0,930364 \\
\hline$-0,808388$ & $-0,460529 i$ & 0,930364 \\
\hline$-0,094422$ & $+0,921898 \mathrm{i}$ & 0,926721 \\
\hline 0,094422 & $-0,921898 \mathrm{i}$ & 0,926721 \\
\hline$-0,924363$ & & 0,924363 \\
\hline 0,202287 & $+0,897041 \mathrm{i}$ & 0,919567 \\
\hline 0,202287 & $-0,897041 i$ & 0,919567 \\
\hline$-0,763643$ & $+0,510049 i$ & 0,918314 \\
\hline$-0,763643$ & $-0,510049 i$ & 0,918314 \\
\hline 0,756818 & $-0,502191 i$ & 0,908279 \\
\hline 0,756818 & $+0,502191 \mathrm{i}$ & 0,908279 \\
\hline$-0,234951$ & $-0,872508 \mathrm{i}$ & 0,903589 \\
\hline$-0,234951$ & $+0,872508 \mathrm{i}$ & 0,903589 \\
\hline 0,876289 & $-0,220286 i$ & 0,903554 \\
\hline 0,876289 & $+0,220286 i$ & 0,903554 \\
\hline$-0,880125$ & $+0,165322 \mathrm{i}$ & 0,895518 \\
\hline$-0,880125$ & $-0,165322 i$ & 0,895518 \\
\hline 0,252501 & $+0,854152 i$ & 0,890692 \\
\hline 0,252501 & $-0,854152 i$ & 0,890692 \\
\hline$-0,078318$ & $-0,886112 i$ & 0,889566 \\
\hline
\end{tabular}


IMPACTOS INDIRECTOS DE LA TASA DE CAMBIO Y LOS PRECIOS DEL PETRÓLEO EN UNA ECONOMÍA NO PETROLERA: APROXIMACIONES VECM Y VAR PARA EL VALLE DEL CAUCA, COLOMBIA

\begin{tabular}{|c|c|c|}
\hline$-0,078318$ & $+0,886112 \mathrm{i}$ & 0,889566 \\
\hline$-0,617414$ & $-0,634148 i$ & 0,885067 \\
\hline$-0,617414$ & $+0,634148 \mathrm{i}$ & 0,885067 \\
\hline$-0,391562$ & $+0,792560 \mathrm{i}$ & 0,884009 \\
\hline$-0,391562$ & $-0,792560 \mathrm{i}$ & 0,884009 \\
\hline$-0,677182$ & $+0,562466 \mathrm{i}$ & 0,880308 \\
\hline$-0,677182$ & $-0,562466 i$ & 0,880308 \\
\hline 0,540066 & $+0,683624 \mathrm{i}$ & 0,871214 \\
\hline 0,540066 & $-0,683624 \mathrm{i}$ & 0,871214 \\
\hline 0,865445 & $-0,087362 i$ & 0,869843 \\
\hline 0,865445 & $+0,087362 i$ & 0,869843 \\
\hline$-0,811249$ & $+0,276738 \mathrm{i}$ & 0,857152 \\
\hline$-0,811249$ & $-0,276738 \mathrm{i}$ & 0,857152 \\
\hline 0,763004 & $-0,384126 i$ & 0,854241 \\
\hline 0,763004 & $+0,384126 \mathrm{i}$ & 0,854241 \\
\hline 0,356029 & $-0,776509 i$ & 0,854238 \\
\hline 0,356029 & $+0,776509 \mathrm{i}$ & 0,854238 \\
\hline$-0,853289$ & & 0,853289 \\
\hline$-0,695396$ & $-0,362201 i$ & 0,784070 \\
\hline$-0,695396$ & $+0,362201 \mathrm{i}$ & 0,784070 \\
\hline 0,578440 & $+0,490899 \mathrm{i}$ & 0,758667 \\
\hline 0,578440 & $-0,490899 i$ & 0,758667 \\
\hline$-0,702431$ & $+0,243924 \mathrm{i}$ & 0,743578 \\
\hline$-0,702431$ & $-0,243924 \mathrm{i}$ & 0,743578 \\
\hline$-0,128825$ & $-0,731188 \mathrm{i}$ & 0,742450 \\
\hline$-0,128825$ & $+0,731188 \mathrm{i}$ & 0,742450 \\
\hline$-0,027761$ & $-0,685491 \mathrm{i}$ & 0,686053 \\
\hline$-0,027761$ & $+0,685491 \mathrm{i}$ & 0,686053 \\
\hline 0,514365 & & 0,514365 \\
\hline$-0,331234$ & $+0,392285 i$ & 0,513424 \\
\hline$-0,331234$ & $-0,392285 i$ & 0,513424 \\
\hline 0,069148 & & 0,069148 \\
\hline
\end{tabular}

VEC specification imposes 5 unit root(s).

Fuente: elaboración del autor. 


\section{Anexo 5. Estadísticos de prueba LM multivariadas para la correlación serial residual hasta el orden especificado}

Hipótesis nula: no hay correlación serial del orden $\mathrm{h}$, la estadística LM está distribuida asintóticamente $\mathrm{x}_{2}$ con $\mathrm{k}_{2}$ grados de libertad.

VAR Residual Serial Correlation LM Te...

Null Hypothesis: no serial correlation at...

Date: 05/22/18 Time: 14:33

Sample: 2001M01 2015M12

Included observations: 166

\begin{tabular}{|c|c|c|}
\hline Lags & LM-Stat & Prob \\
\hline 1 & 35,97154 & 0,4700 \\
\hline 2 & 33,71790 & 0,5776 \\
\hline 3 & 40,61511 & 0,2742 \\
\hline 4 & 42,64507 & 0,2069 \\
\hline 5 & 37,51826 & 0,3994 \\
\hline 6 & 47,29201 & 0,0986 \\
\hline 7 & 34,94754 & 0,5185 \\
\hline 8 & 30,03796 & 0,7472 \\
\hline
\end{tabular}

Probs from chi-square with $36 \mathrm{df}$.

Fuente: elaboración del autor.

\section{Anexo 6. Prueba de heterocedasticidad de White}

Hipótesis nula: sin heterocedasticidad o (sin especificación incorrecta).

VAR Residual Heteroskedasticity Tests: No Cross Terms (only levels and squares)

Date: 05/22/18 Time: 14:34

Sample: 2001M01 2015M12

Included observations: 172

\begin{tabular}{|c|c|c|}
\hline Join test: & \multicolumn{2}{|c|}{} \\
\hline Chi-sq & df & Prob. \\
\hline 2118,780 & 2058 & 0,1714 \\
\hline
\end{tabular}

Fuente: elaboración del autor. 


\section{Anexo 7. Prueba de normalidad de residuales Jarque-Bera model VAR}

Hipótesis nula: los residuos son normales multivariados.

\begin{tabular}{|c|c|c|c|c|}
\hline Component & Kurtosis & Chi-sq & df & Prob. \\
\hline 1 & 3,301441 & 0,651212 & 1 & 0,4197 \\
\hline 2 & 3,343644 & 0,846322 & 1 & 0,3576 \\
\hline 3 & 3,893518 & 5,721679 & 1 & 0,0168 \\
\hline 4 & 3,632808 & 2,869861 & 1 & 0,0903 \\
\hline 5 & 3,572865 & 2,351917 & 1 & 0,1251 \\
\hline 6 & 2,936140 & 0,029226 & 1 & 0,8643 \\
\hline Joint & & 12,47022 & 6 & 0,0523 \\
\hline
\end{tabular}

Fuente: elaboración del autor.

\section{Anexo 8. Raíces del polinomio característico VAR}

Roots of Characteristic Polynomial

Endogenous variables: LOG(PIBVALLED) ISE LOG(E...

Exogenous variables: TASA_DE_INTERES_MUNDIA...

Lag specification: 112

Date: 05/24/18 Time: 11:31

\begin{tabular}{|c|c|c|}
\hline 0,986946 & $-0,020870 \mathrm{i}$ & 0,987167 \\
\hline 0,986946 & $+0,020870 \mathrm{i}$ & 0,987167 \\
\hline 0,974152 & & 0,974152 \\
\hline 0,948446 & & 0,948446 \\
\hline 0,899247 & $-0,179554 \mathrm{i}$ & 0,916998 \\
\hline 0,899247 & $+0,179554 i$ & 0,916998 \\
\hline$-0,764991$ & $+0,476215 i$ & 0,901106 \\
\hline$-0,764991$ & $-0,476215 i$ & 0,901106 \\
\hline$-0,457539$ & $-0,747175 i$ & 0,876135 \\
\hline$-0,457539$ & $+0,747175 \mathrm{i}$ & 0,876135 \\
\hline 0,809296 & $-0,315299 i$ & 0,868547 \\
\hline 0,809296 & $+0,315299 \mathrm{i}$ & 0,868547 \\
\hline 0,355686 & $+0,777739 \mathrm{i}$ & 0,855214 \\
\hline 0,355686 & $-0,777739 i$ & 0,855214 \\
\hline 0,470814 & $+0,709403 \mathrm{i}$ & 0,851422 \\
\hline 0,470814 & $-0,709403 i$ & 0,851422 \\
\hline 0,633064 & $+0,561675 i$ & 0,846315 \\
\hline 0,633064 & $-0,561675 i$ & 0,846315 \\
\hline 0,021282 & $+0,845929 \mathrm{i}$ & 0,846197 \\
\hline 0,021282 & $-0,845929 \mathrm{i}$ & 0,846197 \\
\hline$-0,688318$ & $-0,486363 i$ & 0,842812 \\
\hline$-0,688318$ & $+0,486363 \mathrm{i}$ & 0,842812 \\
\hline$-0,708996$ & $+0,442276 \mathrm{i}$ & 0,835633 \\
\hline$-0,708996$ & $-0,442276 i$ & 0,835633 \\
\hline 0,289186 & $-0,771723 i$ & 0,824126 \\
\hline 0,289186 & $+0,771723 \mathrm{i}$ & 0,824126 \\
\hline 0,681888 & $+0,462512 \mathrm{i}$ & 0,823947 \\
\hline 0,681888 & $-0,462512 i$ & 0,823947 \\
\hline
\end{tabular}




\begin{tabular}{|c|c|c|}
\hline 0,170226 & $+0,787330 i$ & 0,805522 \\
\hline$-0,751419$ & $+0,286552 i$ & 0,804203 \\
\hline$-0,751419$ & $-0,286552 i$ & 0,804203 \\
\hline$-0,147080$ & $-0,784933 i$ & 0,798594 \\
\hline$-0,147080$ & $+0,784933 i$ & 0,798594 \\
\hline$-0,324158$ & $-0,716902 i$ & 0,786782 \\
\hline$-0,324158$ & $+0,716902 i$ & 0,786782 \\
\hline 0,670523 & $-0,303513 i$ & 0,736017 \\
\hline 0,670523 & $+0,303513 i$ & 0,736017 \\
\hline$-0,705637$ & $+0,146584 i$ & 0,720701 \\
\hline$-0,705637$ & $-0,146584 i$ & 0,720701 \\
\hline$-0,713234$ & & 0,713234 \\
\hline$-0,227845$ & $+0,611593 i$ & 0,652656 \\
\hline$-0,227845$ & $-0,611593 i$ & 0,652656 \\
\hline 0,642717 & & 0,642717 \\
\hline 0,175884 & $+0,306104 i$ & 0,353037 \\
\hline 0,175884 & $-0,306104 i$ & 0,353037 \\
\hline$-0,273286$ & & 0,273286 \\
\hline 0,059145 & & 0,059145 \\
\hline
\end{tabular}

No root lies outside the unit circle.

VAR satisfies the stability condition.

Fuente: elaboración del autor. 